\title{
Catalytic Activity Maps for Alloy Nanoparticles
}

\author{
Liang $\mathrm{Cao}^{1,2}$, Tim Mueller ${ }^{2, *}$
}
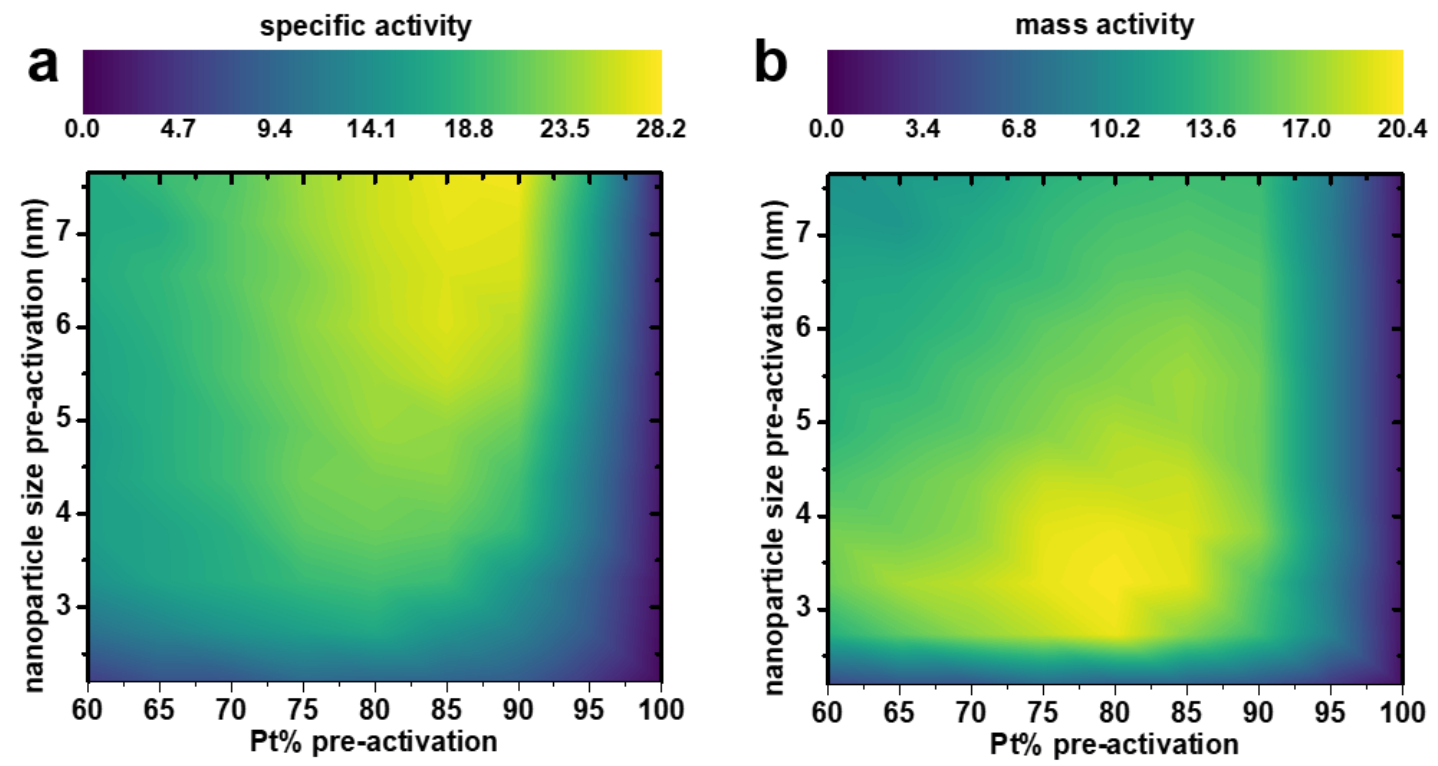

${ }^{1}$ Department of Chemistry, Zhejiang University, Hangzhou, Zhejiang 310000, P. R.

China

${ }^{2}$ Department of Materials Science and Engineering, Johns Hopkins University, Baltimore, Maryland 21218, United States

*Corresponding author(s): Tim Mueller (tmueller@jhu.edu) 


\begin{abstract}
To enable rational design of alloy nanoparticle catalysts, we develop an approach to generate catalytic activity maps of alloy nanoparticles on a grid of particle size and composition. The catalytic activity maps are created by using a quaternary cluster expansion to explicitly predict adsorbate binding energies on alloy nanoparticles of varying shape, size, and atomic order while accounting for interactions among the adsorbates. This cluster expansion is used in kinetic Monte Carlo simulations to predict nanoparticle structures and turnover frequencies on all surface sites. We demonstrate our approach on Pt-Ni octahedral nanoparticle catalysts for the oxygen reduction reaction (ORR), revealing the nanoparticle size and composition predicted to maximize ORR activity.
\end{abstract}

Keywords: rational design, intermetallic, solid-solution, cluster expansion, density functional theory, $\mathrm{Pt}-\mathrm{Ni}$ catalysts, oxygen reduction reaction 


\section{Introduction}

Alloy nanoparticles are well suited to be catalysts due to their high surface to volume ratios and the abundance of ways in which the structures and properties of the catalysts can be tailored. Various experimental synthesis strategies (e.g., core-shell ${ }^{1}$, doping ${ }^{2,3,4}$, shape-engineering ${ }^{5}$ ) have been used to improve the catalytic activity and stability of alloy nanocatalysts, but to limit the number of costly and time consuming experiments that must be done there is great interest in rational nanocatalyst design. Unfortunately, computational design of alloy nanocatalysts remains a significant challenge due to their size and complexity. Density functional theory (DFT) ${ }^{6}$ calculations in standard implementations scale with the number of valence electrons as $\mathrm{O}\left(\mathrm{N}^{3}\right),{ }^{7}$ limiting the diameter of nanoparticles that can be practically modelled to about $2-3 \mathrm{~nm}$, below the typical diameters $(4-10 \mathrm{~nm})$ of particles evaluated experimentally. ${ }^{2,4,5,8,9}$ In addition, the arrangement of atoms in a substitutional alloy can dramatically affect its catalytic properties, ${ }^{10,11}$ making the computational prediction of the atomic structure of the catalyst a critically important step in the design process. Predicting the structure of a substitutional nanoalloy either through thermodynamic $2,12,13,14$ or kinetic ${ }^{3,15,16}$ modelling requires the evaluation of the energies of a large number of candidate structures, where the energy differences between competing alloy structures can be on the order of meV / atom. ${ }^{17}$ The design of alloy nanocatalysts is further complicated by the need to accurately predict adsorbate binding energies, an important descriptor of catalytic activity, on a variety of possible adsorption sites.

Various approaches have been used to computationally predict the structures and properties of alloy nanocatalysts. One strategy is to study nanoparticles that are small enough $(\sim 2 \mathrm{~nm}$ in diameter) to be modeled using DFT. ${ }^{2,18,19,20}$ However the cost of DFT calculations limits the number of structures that can be evaluated this way, and this approach cannot in practice be used 
to model particles of typical experimental sizes. Alternatively, nanoparticle facets may be approximated as extended surfaces, on which adsorbate binding energies can be calculated using DFT. $3,11,21,22$ The disadvantage of this approach is that it does not fully account for the variety of binding sites, including sites near edges and vertices, on a nanoparticle surface.

Due to the cost of directly using DFT, there has recently been significant interest in developing fast and accurate surrogate models for nanocatalysts. Calle-Vallejo et al. ${ }^{23,24}$ developed an approach in which the $* \mathrm{OH}$ and $* \mathrm{OOH}$ adsorption energies on $\mathrm{Pt}$ nanoparticles were linearly correlated with the generalized coordination number $(\mathrm{GCN})$ of the surface binding site, which takes both the first- and second-nearest neighbors into account. Jinnouchi et al. ${ }^{25}$ developed a machine-learned interatomic potential model based on the smooth overlap of atomic positions (SOAP) kernel $^{26}$ to predict structures, catalytic activities, and $\mathrm{N}, \mathrm{O}$, and $\mathrm{NO}$ adsorption energies for fixed-shape $\mathrm{Au}-\mathrm{Rh}$ nanoparticles with varied sizes. For catalysts with structures that can be mapped to a lattice model (e.g., an fcc lattice), formation energies can be accurately calculated using cluster expansions. ${ }^{2}, 3,13,19,20,27$ This approach can be extended to the calculation of adsorption energies by including coordination-number-dependent and metal-specific correction terms into DFT-parametrized cluster expansions ${ }^{3,15}$, or by explicitly including adsorbates in the cluster expansion as a separate species. ${ }^{14}$

Here we present the use of machine-learned cluster expansions to computationally screen nanocatalysts of experimentally relevant sizes and identify those that are expected to have high activity. We demonstrate this approach on $\mathrm{Pt}-\mathrm{Ni}$ nanoparticle catalysts for the oxygen reduction reaction (ORR), which have been extensively studied as promising catalysts in fuel cells. ${ }^{28} \mathrm{We}$ use the adsorption energy of $\mathrm{OH}$ as a descriptor of catalytic activity, as it has been shown to be an accurate descriptor, ${ }^{29,30}$ and in operating conditions $\mathrm{OH}$ is likely to be the most prevalent species 
on the alloy surface. ${ }^{31}$ By explicitly including $\mathrm{OH}$ in the cluster expansion we realistically account for adsorbate-adsorbate interactions (See Methods). Catalytic activity is then predicted using kinetic Monte Carlo (KMC) ${ }^{32,33}$ simulations to calculate the turnover frequencies on all surface sites. Applying this approach to particles over a range of compositions and sizes yields catalytic activity maps for the ORR that indicate the optimal size, composition, and phase of the Pt-Ni nanoparticle catalysts for the ORR, an important step towards the rational design of alloy nanocatalysts.

\section{Results and Discussions}

We start by validating our approach for predicting activities against experimental data. Experimentally, the measured specific and mass activities of $\mathrm{Pt}-\mathrm{Ni}$ nanocatalysts are usually referenced to those of state-of-the-art commercial Pt/C. ${ }^{3,5,15}$ To simulate this reference state, we have calculated size-weighted activity averages according to the diameter length distribution of commercial Pt/C used in our previous work. ${ }^{15}$ Based on the Wulff construction of $\mathrm{Pt}^{14}$, we chose a truncated octahedron as the shape of Pt nanoparticles. Our KMC simulations predict the specific activity of the Pt(111) surface (Supplementary Table 3) to be about 3.8 times relative to that of commercial Pt/C, which is in good agreement with experiments (5 - 10 times). ${ }^{34,35}$ We further validated our approach for predicting catalytic activities by comparing experimentally measured and KMC-predicted activities for two representative Pt-Ni octahedral particles (Table 1). ${ }^{3,15}$ The simulated structures of these particles were matched to experimental data as described in reference. ${ }^{3,15}$ The comparison suggests that our approach slightly overestimates the activities of the $\mathrm{Pt}-\mathrm{Ni}$ nanoparticles, relative to $\mathrm{Pt} / \mathrm{C}$, by a factor of about $1.3-1.7$. The reason for this 
overestimation may be the underestimation of the activity of the Pt/C reference state. More details on the specific activities of the $\mathrm{Pt}(111)$ surface and representative $\mathrm{Pt}-\mathrm{Ni}(111)$ surfaces are provided in the Supplementary Information (Supplementary Table 3, and Supplementary Figs. 2 and 3).

Table 1. Validation of the approach for predicting activities of alloy nanoparticles. Comparison of the experimentally measured and KMC predicted specific and mass activities of two representative Pt-Ni octahedral particles. "before KMC" and "after KMC" mean before and after the simulation of Ni dissolution, respectively. The specific and mass activities were predicted on the snapshots of Pt-Ni particles after KMC (Ni dissolution). The experimental and predicted values are referenced to those of commercial $\mathrm{Pt} / \mathrm{C}$, respectively.

\begin{tabular}{|c|c|c|c|c|c|c|}
\hline \multirow[t]{2}{*}{ References } & \multicolumn{2}{|c|}{ Pt-Ni octahedral particles } & \multicolumn{2}{|c|}{$\begin{array}{l}\text { specific activity } \\
\text { based on } \mathrm{H}_{\text {upd }}\end{array}$} & \multicolumn{2}{|c|}{ mass activity } \\
\hline & before $\mathrm{KMC}$ & after KMC & experiment & prediction & experiment & prediction \\
\hline Jia et al. ${ }^{15}$ & $\mathrm{Pt}_{4495} \mathrm{Ni}_{1680}$ & $\mathrm{Pt}_{4495} \mathrm{Ni}_{895}$ & 10 & 16.90 & 6.88 & 11.03 \\
\hline Cao et $a^{3} .^{3}$ & $\mathrm{Pt}_{4045} \mathrm{Ni}_{2130}$ & $\mathrm{Pt}_{4045} \mathrm{Ni}_{1078}$ & 13.33 & 17.65 & 9.29 & 12.67 \\
\hline
\end{tabular}

To predict the catalytic activity of a nanoparticle it is first necessary to predict the nanoparticle structure. Pt-Ni nanoparticles used as ORR catalysts in proton exchange membrane (PEM) fuel cells typically start as disordered Pt-Ni solid solutions that achieve a Pt-rich shell through an activation process ${ }^{3,15}$ which is usually done by performing cyclic voltammetry (CV) in $\mathrm{N}_{2}$ saturated $0.1 \mathrm{M} \mathrm{HClO}_{4} .{ }^{2,3,15}$ Under such acidic treatment, surface $\mathrm{Ni}$ oxides will dissolve and surface Pt/Ni atoms will migrate. ${ }^{3,15}$ To construct realistic nanoparticle structures, we initialize the particle a fully disordered solid solution and simulate the activation process using kinetic Monte Carlo $(\mathrm{KMC})^{32,33}$, as described in our previous work. ${ }^{3,15} 6175$-atom $(\sim 5.5 \mathrm{~nm})$ nanoparticles with initial (pre-activated) Ni compositions of 40\%, 30\%, $20 \%$ and $10 \%$ lose about 40.0\%, 34.9\%, 31.2\% and $28.0 \%$ of their Ni, respectively (Supplementary Fig. 4). 
Once we have predicted the structure of a nanoparticle, we evaluate catalytic activities by using KMC to estimate the turnover frequency for each adsorbed $\mathrm{OH}$. Specific and mass activities are then calculated by dividing the sum of turnover frequencies by the number of surface Pt atoms and the number of total Pt atoms, respectively. We construct catalytic activity maps (Fig. 1) by repeating this process on a grid with respect to composition and particle edge length (as determined by the pre-activated particles). At each point on this grid, we sample 10 structures to reduce noise introduced by the stochastic determination of the nanoparticle structure. The resulting catalytic activity maps for Pt-Ni octahedral nanoparticles (Fig. 1) reveal that specific activity increases with particle edge length and starts to plateau at an edge length of about $5.5 \mathrm{~nm}$, reaching a maximal value of about 28 times that of $\mathrm{Pt} / \mathrm{C}$ when the initial (pre-activated) nanoparticle has a composition of about $\mathrm{Pt}_{0.85} \mathrm{Ni}_{0.15}$ (Fig. 1a). The mass activity is optimized at a composition of about $\mathrm{Pt}_{0.8} \mathrm{Ni}_{0.2}$ and an edge length of $3.3 \mathrm{~nm}-3.8 \mathrm{~nm}$ (Fig. 1 b). 

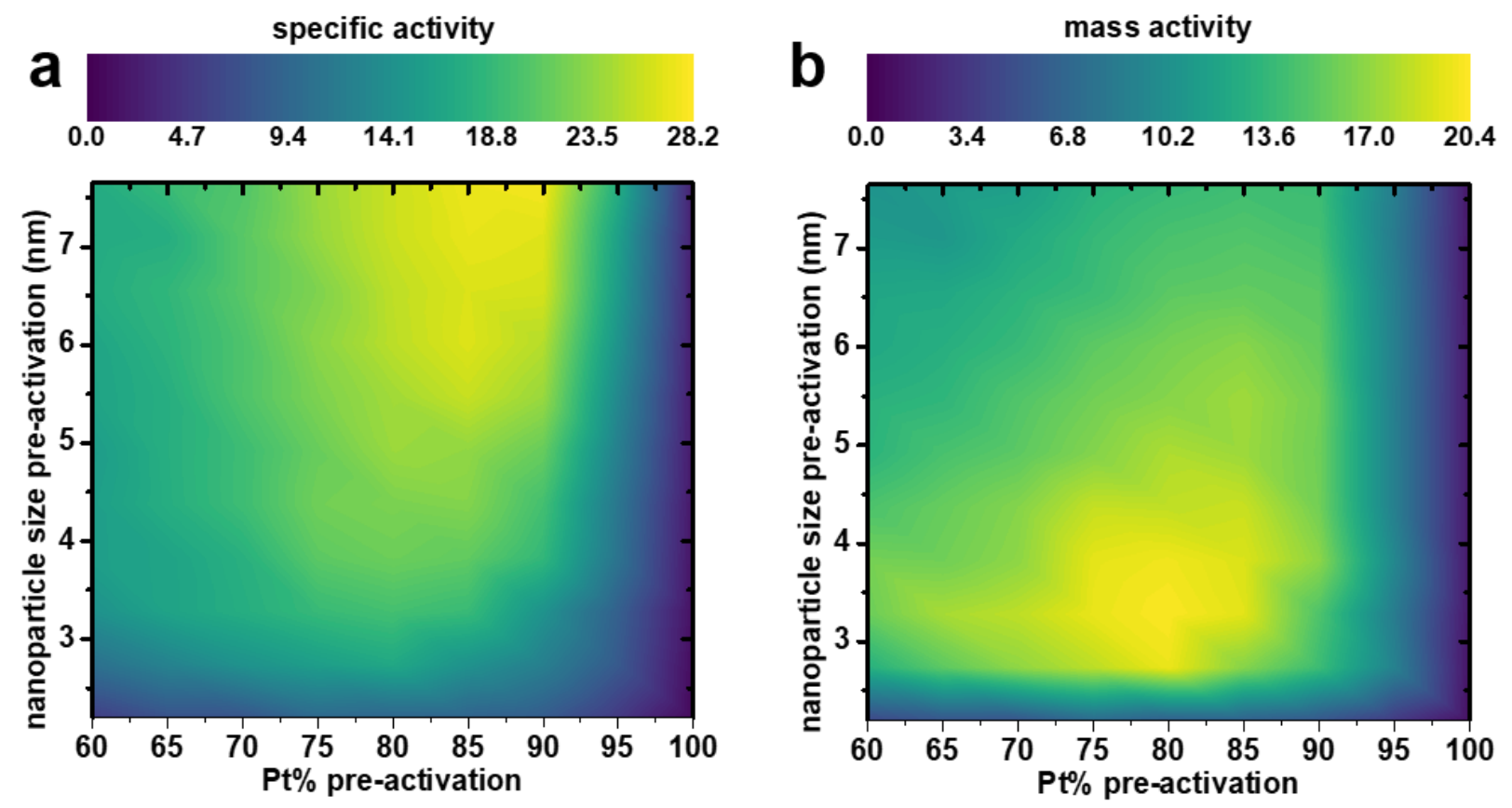

Fig. 1. Size-composition catalytic maps of disordered Pt-Ni nanoparticles. a Predicted specific activity of activated particles. b Predicted mass activity of activated particles. The $\mathrm{x}$-axis is the $\mathrm{Pt}$ composition before KMC (pre-activation) with an increment of 5\%, and the y-axis is the edge length before KMC. All specific and mass activity values are referenced to those of simulated commercial Pt/C (see details in section 5 of the Supplementary Information).

Comparison of our calculations to experimental results is challenging as there have been few systematic experimental studies on size and composition effects in $\mathrm{Pt}-\mathrm{Ni}$ particles. Experimentally, the highest reported mass activity for Pt-Ni nanoparticles by Younan Xia et al. ${ }^{36}$ is about 17 times that of $\mathrm{Pt} / \mathrm{C}$. This result was achieved on octahedral $\mathrm{Pt}_{0.72} \mathrm{Ni}_{0.28}$ particles with an edge length of about $9 \mathrm{~nm}$, but results on other sizes and compositions were not reported in that work. The solvent used in the synthesis of the nanoparticles was found to change the mass activity by a factor of two, highlighting the importance of factors beyond size and composition in determining particle activity. Alonso-Vante et al. studied composition effects in nanoparticles with diameters of 2-3 nm. ${ }^{37}$ The variation of particle size with composition among the samples complicates the analysis of mass activity, but specific activity was found to have a broad peak 
around a composition of about $\mathrm{Pt}_{0.7} \mathrm{Ni}_{0.3}$. This is consistent with our prediction for $3.3 \mathrm{~nm}$ particles, which shows broad peaks with maximal activity at a composition of around $\mathrm{Pt}_{0.8 \mathrm{Ni}} \mathrm{N}_{0.2}$ and only slightly less activity at a composition of $\mathrm{Pt}_{0.7} \mathrm{Ni}_{0.3}$ (Supplementary Fig. 5a). More recently, 4.5-7.5 $\mathrm{nm}$ (in edge length) $\mathrm{Pt}-\mathrm{Ni}$ nanoparticles with a pre-activated composition of $\mathrm{Pt}_{0.6} \mathrm{Ni}_{0.4}$ were reported by Zhang et al. to be more active than particles with a pre-activated composition of $\mathrm{Pt}_{0.75} \mathrm{Ni}_{0.25}{ }^{38}$ The highest mass activity for the $\mathrm{Pt}_{0.6} \mathrm{Ni}_{0.4}$ particles was observed to occur for particles with an edge length of $5.8 \mathrm{~nm}$, whereas the mass activity of the $\mathrm{Pt}_{0.75} \mathrm{Ni}_{0.25}$ nanoparticles showed relatively little change with particle size and was maximized for the largest particles. On the other hand, for pure Pt small nanoparticles $(\sim 2-3 \mathrm{~nm}$ in diameter) have been shown to maximize mass activity. ${ }^{39,40}$

The size and composition at which we predict catalytic activity to be maximized are similar to those observed experimentally, but the experimental results suggest that peak activity occurs for nanoparticles with initial compositions that are more Ni-rich than those predicted by our models. This difference can be partially explained by the amount of Ni dissolution in the particles. For example, Zhang found that $\mathrm{Pt}_{0.6} \mathrm{Ni}_{0.4}$ nanoparticles with an edge length between $4.5 \mathrm{~nm}$ and 8.0 $\mathrm{nm}$ lost about $60 \%$ of the $\mathrm{Ni}$ in the particle after activation, but $\mathrm{Pt}_{0.75} \mathrm{Ni}_{0.25}$ nanoparticles with an edge length between $4.5 \mathrm{~nm}$ and $7.5 \mathrm{~nm}$ only lost about $25 \% .{ }^{38}$ As a result, the post-activated nanoparticles evaluated experimentally had nearly the same composition regardless of their initial compositions. In contrast, for disordered particles with an edge length of $5.5 \mathrm{~nm}$ our simulations predict $40 \% \mathrm{Ni}$ loss for $\mathrm{Pt}_{0.6} \mathrm{Ni}_{0.4}$ and $32 \% \mathrm{Ni}$ loss for $\mathrm{Pt}_{0.75} \mathrm{Ni}_{0.25}$. Thus the post-activated $\mathrm{Pt}_{0.6} \mathrm{Ni}_{0.4}$ nanoparticle has more $\mathrm{Ni}$ than the post-activated $\mathrm{Pt}_{0.75} \mathrm{Ni}_{0.25}$ nanoparticle, which weakens $\mathrm{OH}$ adsorption and leads to lower catalytic activity (Supplementary Fig. 6). Maps of predicted specific 
and mass activities for $\mathrm{Pt}-\mathrm{Ni}$ nanoparticles as a function of the post-activated composition of the nanoparticle are provided in Supplementary Fig. 7.

We have found that in pure Pt and Pt-rich (111) surfaces, density functional theory predicts subsurface vacancies to be significantly more stable than surface vacancies, by about $0.5-1 \mathrm{eV}$ (Supplementary Table 4). We believe this is likely related to reports that DFT significantly underpredicts bulk vacancy formation energies in Pt. ${ }^{41,42}$ Although this effect is less pronounced in the energies calculated using the cluster expansion, the nanoparticle structures generated by our KMC calculations had an unexpectedly high concentration of sub-surface vacancies below flat (111) surfaces ( $0.1 \%$ as shown in Supplementary Fig. 8a). Most of the sub-surface Pt vacancies exist in the $2^{\text {nd }}$ layer of the activated disordered particles. If this result is an artifact of errors inherent in DFT, the resulting over-stabilization of Pt-rich (111) surfaces provides a possible explanation for the apparent under-prediction of $\mathrm{Ni}$ dissolution.

There is evidence that the initial Pt concentration in $\mathrm{Pt}-\mathrm{Ni}$ particles is greater near the particle edges, ${ }^{5,8}$ which may also explain the discrepancy between our predictions and experimental results. To investigate this possibility, we ran simulations on disordered $\mathrm{Pt}-\mathrm{Ni}$ particles initialized with different degrees of Pt-rich edges. This process is described in detail in section 11 of the Supplementary Information. We observed little change in the composition that is predicted to maximize activity (Supplementary Fig. 9), indicating that the initial distribution of Pt and Ni atoms cannot fully explain the slight difference between our calculations and experiments.

The calculated catalytic activity maps provide insights into the atomic origins of catalytic activity trends for alloy nanoparticles. At a composition of $\mathrm{Pt}_{0.85} \mathrm{Ni}_{0.15}$, which is predicted to yield nearly optimal specific activity, the specific activity increases as the edge length increases from 
$2.1 \mathrm{~nm}$ to $5.5 \mathrm{~nm}$, and then starts to plateau when the edge length is larger than $5.5 \mathrm{~nm}$ (Fig. 2a). This behavior can be largely attributed to the fraction of surface Pt atoms that are at sites with a coordination number of 9, denoted as $\operatorname{Pt}(111)$ sites(Fig. 2a). The average turnover frequencies are predicted to be maximized at these sites (Fig. 3b-e and Fig. 4e). The fraction of $\operatorname{Pt}(111)$ sites on the surface reaches a plateau of about 0.72 at an edge length of about $5.5 \mathrm{~nm}(\boldsymbol{F i g} .2 \mathbf{a})$, with the remaining surface Pt atoms on edge sites, vertex sites, near step edges, or near sub-surface vacancies (Fig. 3b). This is in contrast to the expected behavior for an octahedral particle, in which the fraction of (111) sites should increase monotonically with particle size (Fig. 2d). This observation indicates that when the pre-activated edge length gets larger than about $5.5 \mathrm{~nm}$, any size-dependent increase in the density of $\operatorname{Pt}(111)$ sites on the surface is offset by an increase in the density of step edges (or other defects) on the surfaces of activated particles (Supplementary Fig. 10). Other factors, such as the second-layer composition (Supplementary Figs. 10 and 11b, and Supplementary Table 6) are not as well correlated with specific activity. 

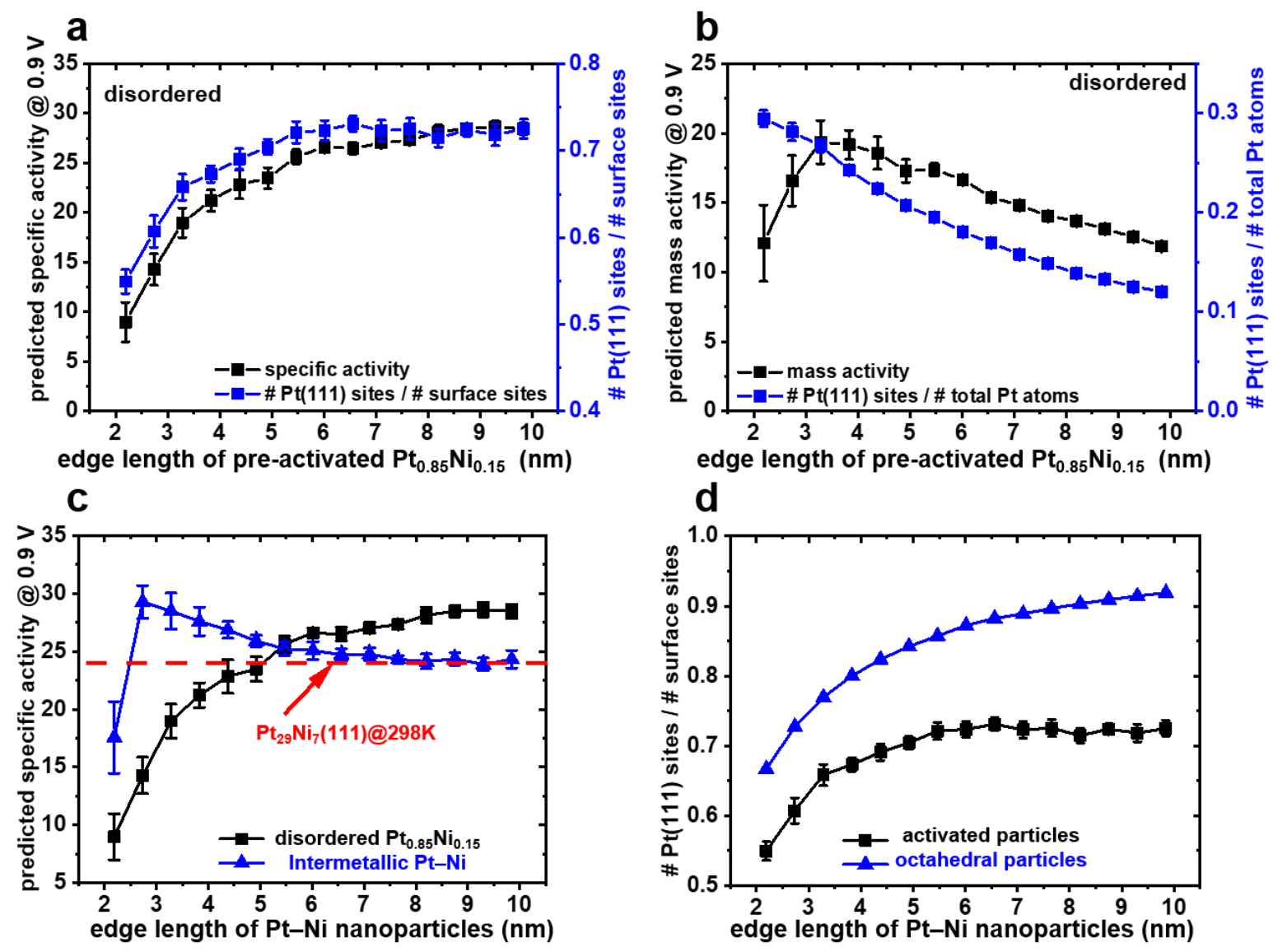

Fig. 2. Size effect on specific and mass activities of Pt-Ni particles. a Predicted specific activity and ratio of the number of surface $\mathrm{Pt}(111)$ sites $(\mathrm{CN}=9)$ to the number of total surface $\mathrm{Pt}$ atoms of disordered $\mathrm{Pt}_{0.85} \mathrm{Ni}_{0.15}$. b Predicted mass activity and ratio of the number of surface $\mathrm{Pt}(111)$ sites $(\mathrm{CN}=9)$ to the number of total $\mathrm{Pt}$ atoms of disordered $\mathrm{Pt}_{0.85} \mathrm{Ni}_{0.15}$. $\mathbf{c}$ Predicted specific activity of disordered $\mathrm{Pt}_{0.85} \mathrm{Ni}_{0.15}$ and intermetallic $\mathrm{Pt}-\mathrm{Ni}$ particles at the $\mathrm{Pt}$ and $\mathrm{Ni}$ chemical potentials that maximize the specific activity of $5.5 \mathrm{~nm}$ particles. The red line represents the $\mathrm{Pt}_{29} \mathrm{Ni}_{7}(111) @ 298 \mathrm{~K}$ surface with $100 \%, 75 \%, 75 \%$, and $75 \%$ in the first, second, third, and fourth layers. ${ }^{12}$ d Ratio of the number of surface $\mathrm{Pt}(111)$ atoms to the number of total surface $\mathrm{Pt}$ atoms of activated disordered $\mathrm{Pt}_{0.85} \mathrm{Ni}_{0.15}$ (labeled as "activated particles") and octahedral particles. All specific activity and mass activity values are referenced to those of simulated commercial $\mathrm{Pt} / \mathrm{C}$. 


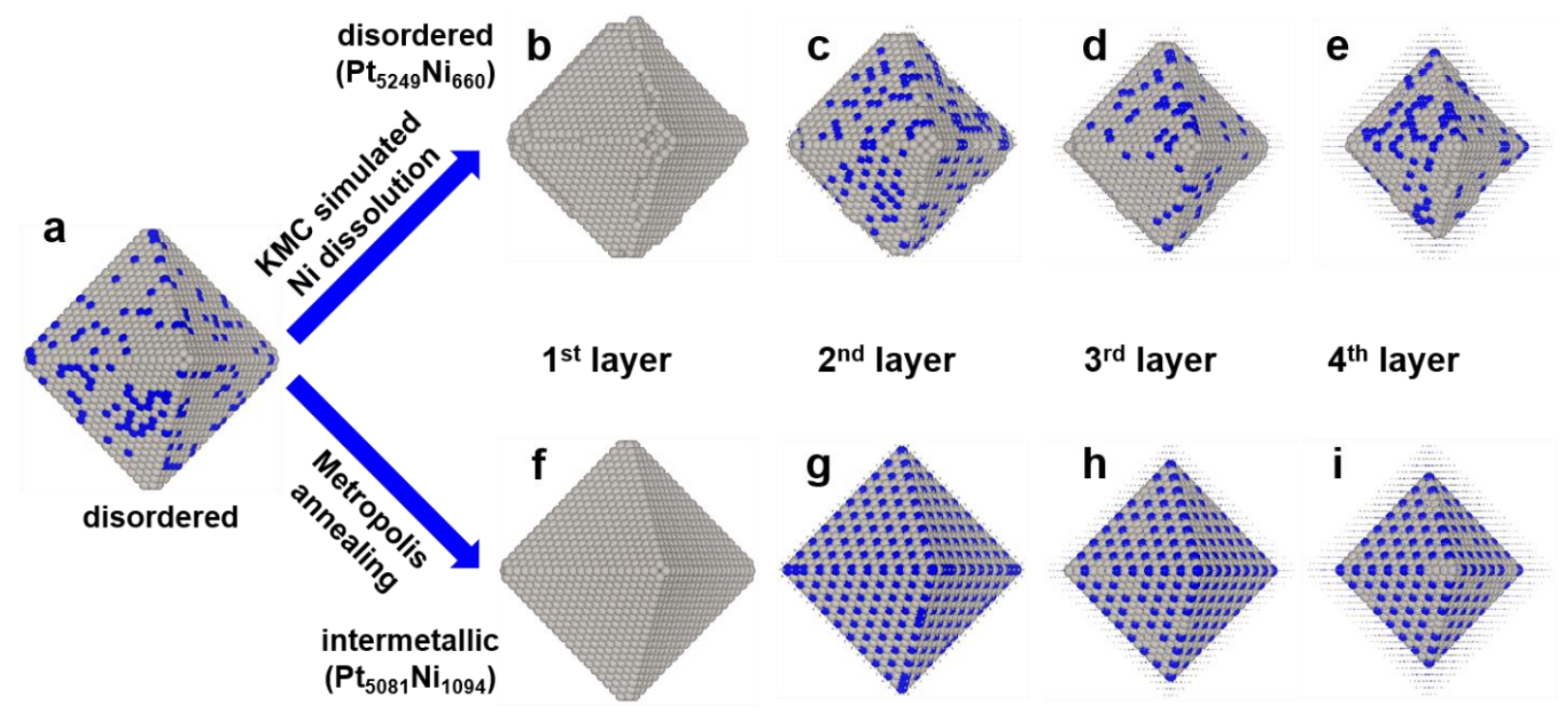

Fig. 3. Layer-by-layer snapshots of disordered and intermetallic Pt-Ni particles. a A snapshot of disordered $\mathrm{Pt}_{0.85} \mathrm{Ni}_{0.15}$ nanoparticle with randomly occupied $\mathrm{Pt} / \mathrm{Ni}$ atoms. b, c, d, e The first (b), second (c), third (d), and fourth (e) layers of a snapshot of activated disordered $\mathrm{Pt}_{0.85} \mathrm{Ni}_{0.15}$ nanoparticle after KMC simulation of Ni dissolution and Pt/Ni migration at $298 \mathrm{~K}$. f, g, h, i The first (f), second (g), third (h), and fourth (i) layers of a snapshot of intermetallic $\mathrm{Pt}_{0.82} \mathrm{Ni}_{0.18}$ nanoparticle after Metropolis Monte Carlo simulation at $298 \mathrm{~K}$.

At the composition of $\mathrm{Pt}_{0.85} \mathrm{Ni}_{0.15}$, mass activity is predicted to increase as the edge length increases from $2.2 \mathrm{~nm}$ to $3.3 \mathrm{~nm}$, and then it decreases as the edge length increases further (Fig. 2b). The low mass activity at small sizes can be explained by the prediction that $\mathrm{OH}$ binding on $\operatorname{Pt}(111)$ sites becomes stronger at small particle sizes (Supplementary Fig. 12), consistent with DFT-calculated results that oxygen binding becomes stronger as the size of nanoparticle size decreases. ${ }^{40}$ At larger sizes, the change in the of $* \mathrm{OH}$ binding energy slows ${ }^{43}$ and the ratio of surface $\mathrm{Pt}(111)$ sites to the number of total $\mathrm{Pt}$ atoms becomes a more important factor in determining the size-dependent mass activity, leading to decreased mass activity (Fig. 2b).

The highest reported specific activity for a Pt-Ni catalyst is on the extended $\mathrm{Pt}_{3} \mathrm{Ni}(111)$ surface ${ }^{31}$, which is likely to be at least partially ordered in the thermodynamically stable $\mathrm{L} 1_{2}$ intermetallic phase. ${ }^{44}$ We have thus also investigated an alternative hypothetical scenario, in which $2.2-9.9 \mathrm{~nm}$ 
octahedral nanoparticles are initialized with thermodynamically stable atomic ordering as determined using Metropolis Monte Carlo simulations ${ }^{45}$ (described in detail in section 14 of the Supplementary Information). The particles are estimated to maintain their octahedral shape due to their thermodynamic stability and the kinetic protection provided by the well-ordered Pt shell; this is supported by the fact that there is almost no Ni dissolution and shape change after running KMC on these intermetallic nanoparticles at $298 \mathrm{~K}$. Within the chemical potential window in which bulk $\mathrm{Pt}_{3} \mathrm{Ni}$ is stable, maximal specific and mass activities are achieved in intermetallic 6175 -atom ( $\sim 5.5 \mathrm{~nm}$ in the edge length) particles with a composition of about $\mathrm{Pt}_{0.82} \mathrm{Ni}_{0.18}$ (Supplementary Fig. 13a). These particles have an $\mathrm{L}_{2}$ core (Fig. 3f-i and Supplementary Figs. 14 and 15) and a Pt monolayer skin, in good agreement with the bulk phase diagram ${ }^{44,46}$ and previous calculations. ${ }^{2}$, ${ }^{13}$ Although intermetallic $\mathrm{Pt}_{0.82} \mathrm{Ni}_{0.18}$ particles and disordered $\mathrm{Pt}_{0.85} \mathrm{Ni}_{0.15}$ nanoparticles both have a Pt skin (Fig. 2b, f), the activities of the intermetallic particles are much more sensitive to the overall particle composition than the activities of the disordered particles, as changes in the composition of the intermetallic particles largely occur through changes in the composition of the near-surface layers (Supplementary Fig.14 and Supplementary Table 7). 

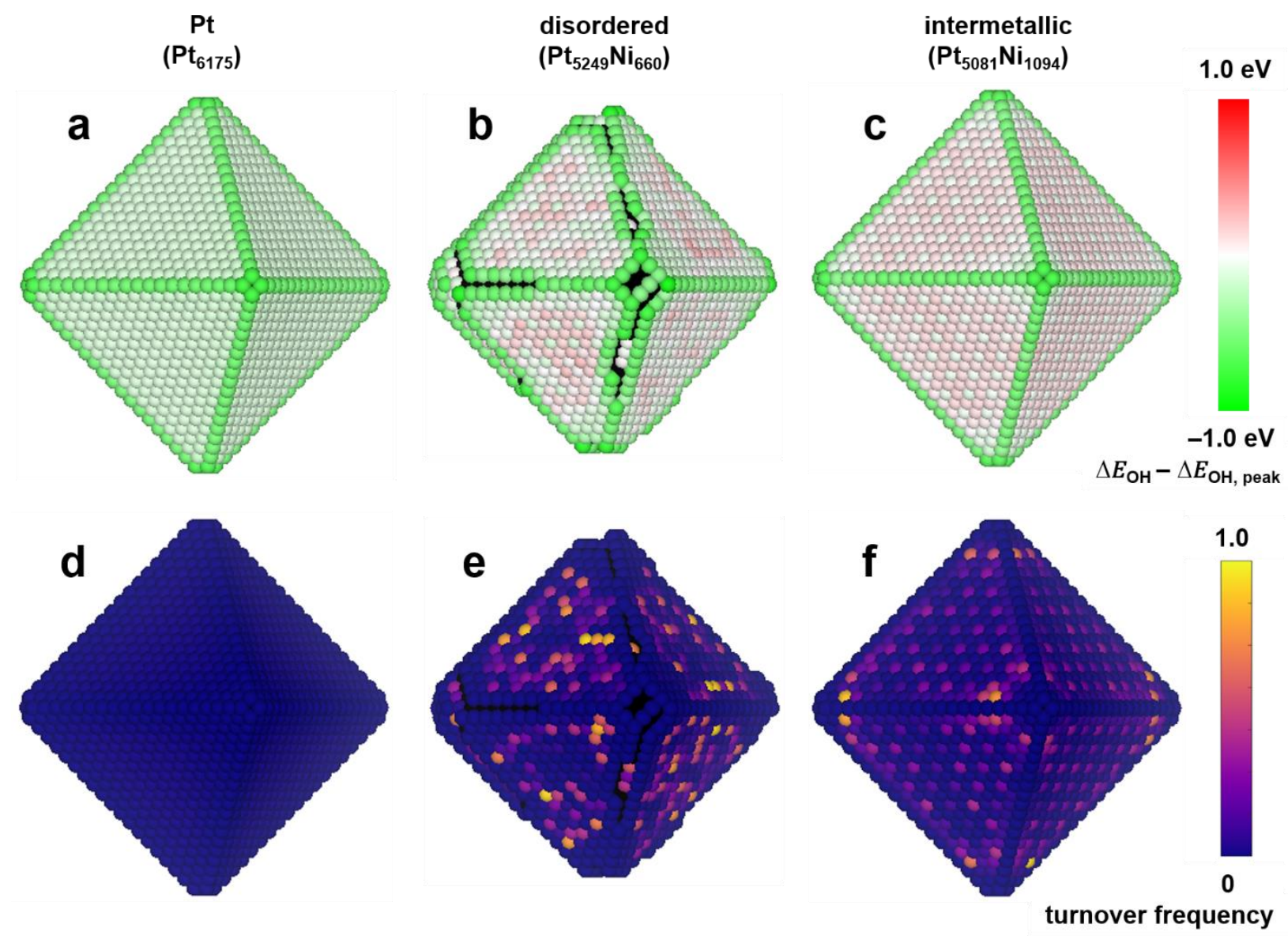

Fig. 4. Predicted *OH binding energy and turnover frequency. a, b, c The average $* \mathrm{OH}$ binding energy on each surface site of three representative nanoparticles with an edge length of $\sim 5.5 \mathrm{~nm}$ : Pt (a), disordered $\mathrm{Pt}_{0.85} \mathrm{Ni}_{0.15}$ in Fig. 3b-e (b), and intermetallic $\mathrm{Pt}_{0.82} \mathrm{Ni}_{0.18}$ in Fig. 3f-i (c). d, e, $\mathbf{f}$ The corresponding average turnover frequency on each site. Binding energies and turnover frequencies referenced to those at the peak of the volcano plot are average values over the number of KMC recording steps, which are 15 times the number of surface Pt atoms. Corresponding histograms of binding energies and turnover frequencies are provided in Supplementary Figs. 19 and 20 .

To investigate the size effect on specific and mass activities of intermetallic particles we set the chemical potential difference between $\mathrm{Pt}$ and Ni to that which maximizes activity in the 6175-atom particles, resulting in an ordered $\mathrm{L}_{2}$ structure at all subsurface layers for particles with edge length 
larger than $3.3 \mathrm{~nm}$ (Fig. 3f-i and Supplementary Fig. 15). For smaller particles, similar atomic structures have been observed but with slightly lower Pt content in sub-surface layers (Supplementary Fig. 16 and Supplementary Table 8). The predicted specific activity and mass activity of intermetallic particles reaches a maximum value at about $2.7 \mathrm{~nm}$ edge length (Fig. $2 \mathrm{c}$ and Supplementary Fig. 17). This peak can be explained by the fact that the average * $\mathrm{OH}$ binding energy on (111) sites for intermetallic particles increases with particle size and crosses over the volcano plot peak at about $2.7 \mathrm{~nm}$ (Supplementary Fig. 18). The predicted specific activity eventually converges to that of the extended $\mathrm{Pt}_{29} \mathrm{Ni}_{7}(111)$ surface, which is predicted to be about 24 times that of commercial Pt/C (Fig. 2c). Thus in the limit of large particle sizes the intermetallic nanoparticles are predicted to be less active than the most active activated disordered particles (Fig. 2c). This observation can be explained by the fact that the second-layer Pt content is predicted to be lower in these intermetallic particles (Supplementary Fig. 16 and Supplementary Table 8) than that in disordered particles (Supplementary Fig. 11 and Supplementary Table 6) due to the dissolution of $\mathrm{Ni}$ atoms from the disordered particles. The relatively Pt-rich second layer in the disordered particles increases the strength of $* \mathrm{OH}$ adsorption and increases catalytic activity. This suggests that the additional stability that may be gained through the synthesis of intermetallic nanoparticles may come at the cost of slightly reduced activity.

\section{Summary and Conclusions}

Computational design of alloy nanocatalysts is challenging due to the need to predict nanoparticle structures, atomic ordering, adsorbate binding energies, adsorbate-adsorbate interactions, and kinetic evolution. We have demonstrated an approach using an accurate surrogate 
model trained on DFT calculations to address all of these challenges, enabling the construction of computationally-generated size-composition activity maps for Pt-Ni nanoparticles for the oxygen reduction reaction. The optimal size and composition predicted by these maps are close to those reported experimentally but slightly more Ni rich (before activation), which may be due to the underprediction of the amount of $\mathrm{Ni}$ that is lost to dissolution. One possible reason for this underprediction is that density functional theory predicts subsurface vacancies on $\operatorname{Pt}(111)$ to be about $1 \mathrm{eV}$ more stable than surface vacancies. This surprising result is likely related to the wellestablished problems DFT has in predicting accurate Pt vacancy formation energies.

Our analysis of site-specific $\mathrm{OH}$ binding energy and turnover frequency on $\mathrm{Pt}-\mathrm{Ni}$ nanoparticles demonstrates that surface sites with low coordination number $(\mathrm{CN}<9)$, such as edge and vertex sites, are highly inactive due to strong $\mathrm{OH}$ binding; while surface $\mathrm{Pt}(111)$ sites $(\mathrm{CN}=9)$ are relatively more active. The fraction of these $\operatorname{Pt}(111)$ sites on the surfaces of activated nanoparticles does not grow as quickly as expected with increasing particle size, likely due to the presence of step edges and other defects. In the limit of large particle sizes, the activated, disordered nanoparticles are still predicted to have slightly higher specific activity than intermetallic $\mathrm{Pt}_{3} \mathrm{Ni}$ with an $\mathrm{L}_{2}$-structured second layer due to lower $\mathrm{Ni}$ content in the second layer. At smaller particle sizes, well-ordered particles may have an advantage as they are predicted to be more resistant to Ni dissolution and thus may be better able to maintain the (111)-rich octahedral shape. However these predictions may not bear out in practice if $\mathrm{Ni}$ dissolution from either the disordered or intermetallic particle is greater than what we have modeled.

The approach we have developed is well suited to study other alloy systems (or multicomponent materials) and other catalytic reactions, especially those for which the binding energies of simple adsorbates have been established as accurate descriptors. The key to modeling these complex 
systems is to develop an accurate surrogate model, which has become increasingly feasible through advances in machine learning. Such models enable researchers to address problems with scale and complexity beyond those achievable with purely ab initio methods but with comparable accuracy, enabling the rational design of alloy nanocatalysts.

\section{Methods}

\section{Density functional theory (DFT) calculations}

DFT calculations were run using the Vienna Ab Initio Simulation Package (VASP) ${ }^{7}$ and the RPBE exchange-correlation functional. ${ }^{47}$ The Ni, Pt_pv_GW, H_GW, and O_GW PBE projectoraugmented wave $(\mathrm{PAW})^{48}$ potentials provided with VASP were used. VASP was run with accurate precision with a plane wave cutoff energy of $434 \mathrm{eV}$. The Brillouin zone was sampled using grids generated by the $k$-point grid server ${ }^{49}$ with a minimum distance of $46.5 \AA$ between real space lattice points. Spin polarization was taken into account in the calculations and the Methfessel-Paxton method $^{50}$ of order 2 was employed to determine electron occupancies with a smearing parameter of $0.2 \mathrm{eV}$. The convergence criteria for the electronic self-consistent iteration and the ionic relaxation loop were set to be $10^{-4} \mathrm{eV}$ and $10^{-3} \mathrm{eV}$, respectively.

\section{Calculation of * $\mathrm{OH}$ binding energies}

The $* \mathrm{OH}$ binding energy $\left(\Delta E_{O H}\right)$ on the surface site, $i$, of a nanoparticle was calculated as

$$
\Delta E_{O H}=E_{D F T}\left(\mathrm{NP}+\mathrm{k}^{*} \mathrm{OH}\right)-E_{D F T}\left(\mathrm{NP}+(\mathrm{k}-1)^{*} \mathrm{OH}\right)+0.5 E_{D F T}\left(\mathrm{H}_{2}\right)-E_{D F T}\left(\mathrm{H}_{2} \mathrm{O}\right),
$$


where $E_{D F T}\left(\mathrm{NP}+\mathrm{n}^{*} \mathrm{OH}\right)$ is the DFT energy of the nanoparticle with $k$ adsorbed $* \mathrm{OH}$, $E_{D F T}\left(\mathrm{NP}+(\mathrm{n}-1)^{*} \mathrm{OH}\right)$ is the DFT energy of the same nanoparticle with one ${ }^{*} \mathrm{OH}$ on site $i$ removed, and $E_{D F T}\left(\mathrm{H}_{2}\right)$ and $E_{D F T}\left(\mathrm{H}_{2} \mathrm{O}\right)$ are the DFT energies of gas-phase $\mathrm{H}_{2}$ and $\mathrm{H}_{2} \mathrm{O}$. By this definition, more negative values of $\Delta E_{O H}$ indicate stronger binding between $* \mathrm{OH}$ and the surface of the nanoparticle.

\section{Cluster expansion construction}

The Pt-Ni-Vacancy cluster expansion included 341 distinct cluster functions and was trained on 201 structures using the Bayesian approach ${ }^{51}$ with a multivariate Gaussian prior distribution, resulting in a leave-one-out cross validation (LOO CV) error of $2.2 \mathrm{meV}$ per atom relative to DFT. To predict $\mathrm{OH}$ binding energies on the surface of $\mathrm{Pt}-\mathrm{Ni}$ nanoparticles with varied sizes, compositions, and shapes we constructed a quaternary Pt-Ni-OH@Pt-Vacancy cluster expansion. As catalytic activities were only calculated on structures that had a Pt skin, we were able to include $\mathrm{OH}$ in the cluster expansion by defining a dummy species representing a $\mathrm{Pt}$ atom bound to $\mathrm{OH}$, which we refer as $\mathrm{OH} @ \mathrm{Pt}$. The Pt-Ni-OH@Pt-Vacancy cluster expansion included 1302 distinct cluster functions and was trained on 353 structures using the Bayesian approach, resulting in a LOO CV error of $2.3 \mathrm{meV}$ per atom relative to DFT. The details of cluster expansion are provided in the Supplementary Information.

\section{KMC algorithm to predict nanoparticle structures}

The Pt-Ni-Vacancy cluster expansion was used in kinetic Monte Carlo (KMC) simulations to predict the atomic structures of Pt-Ni nanoparticles after experimental CV activation. Detailed descriptions of this approach can be found in references 3,15 . 


\section{KMC algorithm to predict ORR activities}

To evaluate the ORR specific activity of $\mathrm{Pt}-\mathrm{Ni}$ nanoparticles, we used a standard "rejectionfree" $\mathrm{KMC}$ algorithm ${ }^{32,33}$ to predict the turnover frequency on each site, here given by the $* \mathrm{OH}$ desorption rate. For each KMC step, the only allowed events were adsorption and desorption. We did not model surface diffusion, which should not affect the equilibrium $* \mathrm{OH}$ coverage and distribution. Only atoms with coordination numbers larger than 3 and smaller than 10 were considered to be surface atoms. The transition rate for each event is calculated as $e^{\frac{-\mathrm{E}_{a}}{\mathrm{kT}}}$, where $\mathrm{E}_{a}$ is the activation free energy for $\mathrm{OH}$ to desorb or adsorb. The free energy for adsorption was calculated as a function of the $* \mathrm{OH}$ binding energy to ensure that the turnover frequency was consistent with the right (adsorption-limited) leg of the Sabatier volcano as determined by the work

of Norskov et al. ${ }^{52,53}$, and the free energy for desorption was similarly calculated to be consistent with the left (desorption-limited) leg. Additional details are provided in the Supplementary Information.

\section{ASSOCIATED CONTENT}

\section{Supplementary Information}

Theoretical details of the cluster expansion, kinetic Monte Carlo (KMC) simulations to predict ORR activities, Metropolis Mote Carlo simulations, and other supplementary figures and tables are given. 


\section{AUTHOR INFORMATION}

\section{Corresponding Author}

*tmueller@jhu.edu

\section{Author Contributions}

The manuscript was written through contributions of all authors.

\section{Notes}

The authors declare no competing financial interests.

\section{ACKNOWLEDGMENT}

Liang Cao and Tim Mueller acknowledge support from the Office of Naval Research under MURI award N00014-15-1-2681. Computational resources were provided by XSEDE through award DMR-140068 and by the Maryland Advanced Research Computing Center (MARCC). Atomicscale structural images were generated using VESTA. ${ }^{54}$ 


\section{REFERENCES}

1. Kang Y, Snyder J, Chi M, Li D, More KL, Markovic NM, et al. Multimetallic Core/Interlayer/Shell Nanostructures as Advanced Electrocatalysts. Nano Lett 2014, 14(11): 6361-6367.

2. Huang X, Zhao Z, Cao L, Chen Y, Zhu E, Lin Z, et al. High-performance transition metal-doped Pt3Ni octahedra for oxygen reduction reaction. Science 2015, 348(6240): 1230-1234.

3. Cao L, Zhao Z, Liu Z, Gao W, Dai S, Gha J, et al. Differential Surface Elemental Distribution Leads to Significantly Enhanced Stability of PtNi-Based ORR Catalysts. Matter 2019, 1(6): 1567-1580.

4. Beermann V, Gocyla M, Willinger E, Rudi S, Heggen M, Dunin-Borkowski RE, et al. Rh-Doped Pt-Ni Octahedral Nanoparticles: Understanding the Correlation between Elemental Distribution, Oxygen Reduction Reaction, and Shape Stability. Nano Lett 2016, 16(3): 1719-1725.

5. Cui C, Gan L, Heggen M, Rudi S, Strasser P. Compositional Segregation in Shaped Pt Alloy Nanoparticles and Their Structural Behaviour during Electrocatalysis. Nat Mater 2013, 12(8): 765-771.

6. Kohn W, Sham LJ. Self-Consistent Equations Including Exchange and Correlation Effects. Phys Rev 1965, 140(4A): A1133-A1138.

7. Kresse G, Furthmueller J. Efficient Iterative Schemes for Ab initio Total-Energy Calculations Using a Plane-Wave Basis Set. Phys Rev B 1996, 54: 11169-11186.

8. Gan L, Cui C, Heggen M, Dionigi F, Rudi S, Strasser P. Element-Specific Anisotropic Growth of Shaped Platinum Alloy Nanocrystals. Science 2014, 346(6216): 1502-1506.

9. Beermann V, Kühl S, Strasser P. Tuning the Catalytic Oxygen Reduction Reaction Performance of Pt-Ni Octahedral Nanoparticles by Acid Treatments and Thermal Annealing. J Electrochem Soc 2018, 165(15): J3026-J3030.

10. Chen M, Kumar D, Yi C-W, Goodman DW. The Promotional Effect of Gold in Catalysis by Palladium-Gold. Science 2005, 310(5746): 291-293. 
11. Wang Y, Cao L, Libretto NJ, Li X, Li C, Wan Y, et al. Ensemble Effect in Bimetallic Electrocatalysts for CO2 Reduction. J Am Chem Soc 2019, 141(42): 16635-16642.

12. Cao L, Mueller T. Rational Design of $\mathrm{Pt}_{3} \mathrm{Ni}$ Surface Structures for the Oxygen Reduction Reaction. J Phys Chem C 2015, 119(31): 17735-17747.

13. Cao L, Mueller T. Theoretical Insights into the Effects of Oxidation and Mo-Doping on the Structure and Stability of Pt-Ni Nanoparticles Nano Lett 2016, 16(12): 7748-7754.

14. Li C, Raciti D, Pu T, Cao L, He C, Wang C, et al. Improved Prediction of Nanoalloy Structures by the Explicit Inclusion of Adsorbates in Cluster Expansions. J Phys Chem C 2018, 122(31): 18040-18047.

15. Jia Q, Zhao Z, Cao L, Li J, Ghoshal S, Davies V, et al. Roles of Mo Surface Dopants in Enhancing the ORR Performance of Octahedral PtNi Nanoparticles. Nano Lett 2018, 18(2): 798-804.

16. Li C, Nilson T, Cao L, Mueller T. Predicting activation energies for vacancy-mediated diffusion in alloys using a transition-state cluster expansion. Phys Rev Mater 2021, in press.

17. Curtarolo S, Morgan D, Ceder G. Accuracy of ab initio methods in predicting the crystal structures of metals: A review of 80 binary alloys. Calphad 2005, 29(3): 163-211.

18. Verga LG, Aarons J, Sarwar M, Thompsett D, Russell AE, Skylaris CK. DFT calculation of oxygen adsorption on platinum nanoparticles: coverage and size effects. Faraday Discussions 2018, 208(0): 497-522.

19. Tan TL, Wang L-L, Johnson DD, Bai K. A Comprehensive Search for Stable Pt-Pd Nanoalloy Configurations and Their Use as Tunable Catalysts. Nano Lett 2012, 12(Copyright (C) 2015 American Chemical Society (ACS). All Rights Reserved.): 48754880 .

20. Yuge K. Segregation of $\mathrm{Pt}_{28} \mathrm{Rh}_{27}$ Bimetallic Nanoparticles: A First-Principles Study. $J$ Phys: Condens Matter 2010, 22(24): 245401. 
21. Wang X, Wang Z, Zhuang T-T, Dinh C-T, Li J, Nam D-H, et al. Efficient upgrading of CO to C3 fuel using asymmetric C-C coupling active sites. Nat Commun 2019, 10(1): 5186.

22. Chou S-W, Lai Y-R, Yang YY, Tang C-Y, Hayashi M, Chen H-C, et al. Uniform size and composition tuning of $\mathrm{PtNi}$ octahedra for systematic studies of oxygen reduction reactions. J Catal 2014, 309(0): 343-350.

23. Calle-Vallejo F, Tymoczko J, Colic V, Vu QH, Pohl MD, Morgenstern K, et al. Finding optimal surface sites on heterogeneous catalysts by counting nearest neighbors. Science 2015, 350(6257): 185.

24. Calle-Vallejo F, Martínez JI, García-Lastra JM, Sautet P, Loffreda D. Fast Prediction of Adsorption Properties for Platinum Nanocatalysts with Generalized Coordination Numbers. Angew Chem, Int Ed 2014, 53(32): 8316-8319.

25. Jinnouchi R, Asahi R. Predicting Catalytic Activity of Nanoparticles by a DFT-Aided Machine-Learning Algorithm. J Phys Chem Lett 2017, 8(17): 4279-4283.

26. Bartók AP, Kondor R, Csányi G. On representing chemical environments. Phys Rev B 2013, 87(18): 184115.

27. Sanchez JM, Ducastelle F, Gratias D. Generalized Cluster Description of Multicomponent Systems. Phys A (Amsterdam, Neth) 1984, 128(1-2): 334-350.

28. Zhao Z, Chen C, Liu Z, Huang J, Wu M, Liu H, et al. Pt-Based Nanocrystal for Electrocatalytic Oxygen Reduction. Adv Mater 2019, 0(0): 1808115.

29. Viswanathan V, Hansen HA, Rossmeisl J, Nørskov JK. Unifying the 2e- and 4eReduction of Oxygen on Metal Surfaces. J Phys Chem Lett 2012, 3(20): 2948-2951.

30. Viswanathan V, Hansen HA, Rossmeisl J, Nørskov JK. Universality in Oxygen Reduction Electrocatalysis on Metal Surfaces. ACS Catal 2012, 2(8): 1654-1660.

31. Stamenkovic VR, Fowler B, Mun BS, Wang G, Ross PN, Lucas CA, et al. Improved Oxygen Reduction Activity on $\mathrm{Pt}_{3} \mathrm{Ni}(111)$ via Increased Surface Site Availability. Science 2007, 315: 493-497. 
32. Schulze TP. Efficient kinetic Monte Carlo simulation. J Comput Phys 2008, 227(4): 2455-2462.

33. Serebrinsky SA. Physical Time Scale in Kinetic Monte Carlo Simulations of ContinuousTime Markov Chains. Phys Rev E 2011, 83(3): 037701.

34. Gasteiger HA, Kocha SS, Sompalli B, Wagner FT. Activity Benchmarks and Requirements for Pt, Pt-Alloy, and non-Pt Oxygen Reduction Catalysts for PEMFCs. Appl Catal, B 2005, 56(Copyright (C) 2015 American Chemical Society (ACS). All Rights Reserved.): 9-35.

35. Paulus UA, Wokaun A, Scherer GG, Schmidt TJ, Stamenkovic V, Radmilovic V, et al. Oxygen Reduction on Carbon-Supported $\mathrm{Pt}-\mathrm{Ni}$ and $\mathrm{Pt}-\mathrm{Co}$ Alloy Catalysts. J Phys Chem B 2002, 106(16): 4181-4191.

36. Choi S-I, Xie S, Shao M, Odell JH, Lu N, Peng H-C, et al. Synthesis and Characterization of $9 \mathrm{~nm}$ Pt-Ni Octahedra with a Record High Activity of $3.3 \mathrm{~A} / \mathrm{mgPt}$ for the Oxygen Reduction Reaction. Nano Lett 2013, 13(7): 3420-3425.

37. Yang H, Vogel W, Lamy C, Alonso-Vante N. Structure and Electrocatalytic Activity of Carbon-Supported Pt-Ni Alloy Nanoparticles Toward the Oxygen Reduction Reaction. $J$ Phys Chem B 2004, 108(30): 11024-11034.

38. Zhang C, Hwang SY, Peng Z. Size-dependent oxygen reduction property of octahedral Pt-Ni nanoparticle electrocatalysts. Journal of Materials Chemistry A 2014, 2(46): 19778-19787.

39. Perez-Alonso FJ, McCarthy DN, Nierhoff A, Hernandez-Fernandez P, Strebel C, Stephens IEL, et al. The Effect of Size on the Oxygen Electroreduction Activity of MassSelected Platinum Nanoparticles. Angew Chem, Int Ed 2012, 51(19): 4641-4643.

40. Shao M, Peles A, Shoemaker K. Electrocatalysis on Platinum Nanoparticles: Particle Size Effect on Oxygen Reduction Reaction Activity. Nano Lett 2011, 11(9): 3714-3719.

41. Mattsson TR, Mattsson AE. Calculating the vacancy formation energy in metals: Pt, Pd, and Mo. Phys Rev B 2002, 66(21): 214110. 
42. Nazarov R, Hickel T, Neugebauer J. Vacancy formation energies in fcc metals: Influence of exchange-correlation functionals and correction schemes. Phys Rev B 2012, 85(14): 144118.

43. Leontyev IN, Kuriganova AB, Leontyev NG, Hennet L, Rakhmatullin A, Smirnova NV, et al. Size dependence of the lattice parameters of carbon supported platinum nanoparticles: X-ray diffraction analysis and theoretical considerations. RSC Adv 2014, 4(68): 35959-35965.

44. Cao L, Niu L, Mueller T. Computationally generated maps of surface structures and catalytic activities for alloy phase diagrams. Proc Natl Acad Sci U S A 2019, 116(44): 22044-22051.

45. Metropolis N, Rosenbluth AW, Rosenbluth MN, Teller AH, Teller E. Equation of State Calculations by Fast Computing Machines. J Chem Phys 1953, 21(Copyright (C) 2015 American Chemical Society (ACS). All Rights Reserved.): 1087-1092.

46. Lu X-G, Sundman B, A ${ }^{\circ}$ gren J. Thermodynamic assessments of the Ni-Pt and Al-Ni-Pt systems. Calphad 2009, 33(3): 450-456.

47. Hammer B, Hansen LB, Nørskov JK. Improved adsorption energetics within densityfunctional theory using revised Perdew-Burke-Ernzerhof functionals. Physical Review B 1999, 59(11): 7413.

48. Blochl PE. Projector Augmented-Wave Method. Phys Rev B 1994, 50(24): 17953-17979.

49. Wisesa P, McGill KA, Mueller T. Efficient Generation of Generalized Monkhorst-Pack Grids through the Use of Informatics. Phys Rev B 2016, 93(15): 155109.

50. Methfessel M, Paxton AT. High-Precision Sampling for Brillouin-Zone Integration in Metals. Phys Rev B 1989, 40(6): 3616-3621.

51. Mueller T, Ceder G. Bayesian Approach to Cluster Expansions. Phys Rev B 2009, 80: 024103.

52. Rossmeisl J, Logadottir A, Norskov JK. Electrolysis of Water on (Oxidized) Metal Surfaces. Chem Phys 2005, 319(Copyright (C) 2015 American Chemical Society (ACS). All Rights Reserved.): 178-184. 
53. Karlberg GS, Rossmeisl J, Norskov JK. Estimations of Electric Field Effects on the Oxygen Reduction Reaction based on the Density Functional Theory. Phys Chem Chem Phys 2007, 9: 5158-5161.

54. Momma K, Izumi F. VESTA: A Three-Dimensional Visualization System for Electronic and Structural Analysis. J Appl Crystallogr 2008, 41: 653-658. 


\title{
Supplementary Information
}

\section{Catalytic Activity Maps for Alloy Nanoparticles}

\author{
Liang $\mathrm{Cao}^{1,2}$, Tim Mueller ${ }^{2, *}$ \\ ${ }^{1}$ Department of Chemistry, Zhejiang University, Hangzhou, Zhejiang 310000, P. R. China \\ ${ }^{2}$ Department of Materials Science and Engineering, Johns Hopkins University, Baltimore, \\ Maryland 21218, United States
}




\section{Cluster expansion}

Cluster expansions are generalized Ising models, ${ }^{1}$ in which the "spin" variables assigned to each site in an Ising model are replaced by "site" variables that indicate which species (or vacancy) is present at each site. ${ }^{2,3}$ A property of the material (e.g., formation energy) can be expressed as a function of these site variables:

$$
F(\mathbf{s})=V_{0}+\sum_{\text {clusters }} V_{\text {cluster }} \prod_{i \in \text { cluster }} \Theta_{b, \mathrm{i}}\left(s_{i}\right)
$$

where the unknown coefficients, $V_{\text {cluster }}$, are known as effective cluster interactions (ECIs), $s_{i}$ is the site variable at the $i^{\text {th }}$ site, $\Theta_{b, i}$ is the $b^{\text {th }}$ basis function for the $i^{\text {th }}$ site, and $V_{0}$ is a constant term representing the ECI for the "empty" cluster. The sum is over clusters of sites, where the number of sites in a cluster may range from 1 to all the sites in the material. When all possible clusters are included in the expansion, the expansion in Eq. [1] is exact. In practice, the ECIs for clusters that contain a large number of sites or sites that are far apart are usually negligible, allowing for the expansion to be truncated to a sum with a finite number of ECIs with little loss of accuracy.

To calculate the ORR activities on Pt-Ni nanoparticles, two cluster expansions models have been developed. The first one is a quaternary Pt-Ni-OH@Pt-Vacancy cluster expansion, which is used to explicitly predict $* \mathrm{OH}$ binding energies on the nanoparticle surface and account for the interactions among adsorbed $* \mathrm{OH}$. The second one is the Pt$\mathrm{Ni}-$ Vacancy cluster expansion, which is used to simulate the structural evolution of $\mathrm{Pt}-\mathrm{Ni}$ nanoparticles using kinetic Monte Carlo and to predict the thermodynamically stable nanoparticles using Metropolis Monte Carlo. 


\subsection{Pt-Ni-OH@Pt-Vacancy cluster expansion on nanoparticles}

The Pt-Ni-OH@Pt-Vacancy cluster expansion was built using an approach similar to one we previously used to build a Pt-Ni-Mo-Vacancy cluster expansion. ${ }^{4,5,6}$ In the present work, we consider a surface Pt atom and a hydroxyl $(\mathrm{OH})$ adsorbed on its top site as a single dummy species, which is expressed as*OH@Pt. The quaternary Pt-Ni-OH@PtVacancy cluster expansion was generated on an fcc lattice in which each site could be occupied by*OH@Pt, nickel $(\mathrm{Ni})$, platinum $(\mathrm{Pt})$, or a vacancy. Site variable values of 0 , 1,2 , and 3 respectively were assigned to these species. The constraint for the dummy species*OH@Pt is that it only can occupy surface sites. For each nanoparticle, atoms with coordination number (nearest-neighboring metal sites) larger than 2 and smaller than 10 are considered to be on surface sites. Pt and Ni Atoms were not permitted to have fewer than 3 nearest neighbors in Monte Carlo simulations. A discrete cosine basis was used to generate the cluster functions, where the $b^{\text {th }}$ basis function of the site variable $s$ is given by

$$
\Theta_{b}=\left\{\begin{array}{cc}
1 & \text { for } \mathrm{b}=0 \\
\sqrt{2} \cos (\pi b(2 \mathrm{~s}+1) / 8) & \text { for } \mathrm{b}>0
\end{array},\right.
$$

for $b \in\{0,1,2,3\}$.

For the generation of training data, we reused all training structures that do not contain Mo atoms from the Pt-Ni-Mo-Vacancy cluster expansion ${ }^{5}$, leading to an initial set of 151 random clean Pt-Ni particles. By randomly selecting from the pool of 151 clean particles, we created additional 95 random $\mathrm{Pt}-\mathrm{Ni}$ particles with randomly decorated $* \mathrm{OH}$ on surface Pt atoms at varied coverages (35 particles with $0.4 \mathrm{ML}, 44$ particles with $0.2 \mathrm{ML}, 8$ particles with $0.15 \mathrm{ML}$, and 8 particles with 0.1 ML, respectively). All nanoparticles were generated under the constraint that there had to be more than 100 total $\mathrm{Pt} / \mathrm{Ni}$ atoms in the nanoparticle, as we have found that the inclusion of smaller particles can lead to cluster expansions with poor predictive accuracy for multi-nanometer nanoparticles (probably due to quantum size effects). Nanoparticles that experienced significant reconstruction upon relaxation, defined as an atom traveling more than $75 \%$ the nearest-neighbor distance from its initial site, were excluded. All nanoparticles were contained in a cubic cell with a lattice parameter of 28.8 
$\AA$. The resulting set of random nanoparticles included 151 clean Pt-Ni nanoparticles without $* \mathrm{OH}$ and $95 \mathrm{Pt}-\mathrm{Ni}$ nanoparticles with $* \mathrm{OH}$. To improve the accuracy of predicted ORR activities, we included 22 additional $\mathrm{Pt}-\mathrm{Ni}$ nanoparticles with varied coverages and patterns of adsorbed $* \mathrm{OH}$ determined by KMC simulations of ORR activities (see details in section 2) and 7 9-layer $\mathrm{Pt}-\mathrm{Ni}(111)$ surfaces into the training set.

In addition to these structures, the training data consisted of the pure elements $\mathrm{Ni}$, and $\mathrm{Pt}$ in a bulk fcc crystal, vacuum (a lattice containing only vacant sites), and various lowenergy structures predicted over the course of this research, for a total of 353 unique structures. These 353 unique training structures included 198 clean Pt-Ni nanoparticles, 145 Pt-Ni nanoparticles with *OH, 7 9-layer Pt-Ni(111) surfaces, 1 Pt bulk, 1 Ni bulk, and 1 vacuum structure. To reduce the prediction error of the cluster expansion ${ }^{7}$, the pure elements and vacuum were included twice in the training set. All energies of training set structures were calculated using density functional theory (DFT). Details of the DFT calculations are provided in the Methods section of main body. The effective cluster interactions (ECIs) of the cluster expansions were fit to the DFT-calculated formation energies of fully relaxed nanoparticles relative to the reference states of bulk fcc $\mathrm{Ni}$, bulk fcc $\mathrm{Pt}$, and $0.5 \mathrm{E}\left(\mathrm{H}_{2}\right)-\mathrm{E}\left(\mathrm{H}_{2} \mathrm{O}\right)$ for $\mathrm{OH}$. The formation energy can be expressed as

$$
\begin{aligned}
& F E_{D F T}\left(\mathrm{Pt}_{\mathrm{m}} \mathrm{Ni}_{\mathrm{n}}{ }^{*} \mathrm{OH}_{\mathrm{k}}\right) \\
& =E_{D F T}\left(\mathrm{Pt}_{\mathrm{m}} \mathrm{Ni}_{\mathrm{n}}{ }^{*} \mathrm{OH}_{\mathrm{k}}\right)-m E_{D F T}(\mathrm{Pt})-n E_{D F T}(\mathrm{Ni})+k\left[0.5 E_{D F T}\left(\mathrm{H}_{2}\right)-E_{D F T}\left(\mathrm{H}_{2} \mathrm{O}\right)\right],
\end{aligned}
$$

where $E_{D F T}\left(\mathrm{Pt}_{\mathrm{m}} \mathrm{Ni}_{\mathrm{n}}{ }^{*} \mathrm{OH}_{\mathrm{k}}\right)$ is the total DFT energy of the Pt-Ni nanoparticle $\left(\mathrm{Pt}_{\mathrm{m}} \mathrm{Ni}_{\mathrm{n}}\right)$ with k adsorbed $* \mathrm{OH}, E_{D F T}(\mathrm{Pt})$ and $E_{D F T}(\mathrm{Ni})$ are the DFT energies of bulk fcc Pt and Ni per atom, and $E_{D F T}\left(\mathrm{H}_{2}\right)$ and $E_{D F T}\left(\mathrm{H}_{2} \mathrm{O}\right)$ are the DFT energies of gas-phase $\mathrm{H}_{2}$ and $\mathrm{H}_{2} \mathrm{O}$. Our choice of $\mathrm{H}_{2}(\mathrm{~g})$ and $\mathrm{H}_{2} \mathrm{O}(\mathrm{g})$ as reference states avoids potential errors in the calculation of the energy of $\mathrm{O}_{2}(\mathrm{~g})$ and allows for more direct comparisons with previous calculations. ${ }^{8}$

The Pt-Ni-OH@Pt-Vacancy cluster expansion was truncated to include the empty cluster, the one-body (point) cluster, all 2-body clusters up to the seventh-nearest neighbor, all 3-body clusters up to the fifth-nearest neighbor, all 4-body clusters up to the third- 
nearest neighbor, and 5-, and 6-body clusters up to the second-nearest neighbor, for a total of 1302 symmetrically distinct cluster functions. The ECIs for these cluster functions were fit to the training data using the Bayesian approach with a multivariate Gaussian prior distribution. ${ }^{9}$ The inverse of the covariance matrix for the prior, $\Lambda$, was diagonal, with elements given by

$$
\lambda_{\alpha \alpha}=\left\{\begin{array}{cc}
0 & \text { for } \mathrm{n}_{\alpha}=0 \\
\lambda_{1} & \text { for } \mathrm{n}_{\alpha}=1 \\
\lambda_{2}\left(1+r_{\alpha}\right)^{\lambda_{3}} e^{\lambda_{4} n_{\alpha}} & \text { for } \mathrm{n}_{\alpha}>1
\end{array}\right.
$$

where $\mathrm{n}_{\alpha}$ is the number of sites in cluster function $\alpha, r_{\alpha}$ is the maximum distance between sites, and the parameters $\lambda_{1}, \lambda_{2}, \lambda_{3}$, and $\lambda_{4}$ were determined by using a conjugate gradient algorithm to minimize the root mean square leave-one-out cross validation (RMS LOOCV) score, an estimate of prediction error. ${ }^{10}$ The final values for these parameters were $1.000 \times 10^{-8}, 9.414 \times 10^{-12}, 4.286$, and 2.986 respectively. The resulting cluster expansion had a RMS LOOCV error of $2.3 \mathrm{meV}$ per atom relative to DFT calculations. 


\subsection{Pt-Ni-Vacancy cluster expansion on nanoparticles}

The Pt-Ni-Vacancy cluster expansion was built by removing the dummy species (*OH@Pt: surface Pt atom adsorbed with *OH) from $\mathrm{Pt}-\mathrm{Ni}-\mathrm{OH}-\mathrm{Vacancy}$ cluster expansion, removing the training structures containing adsorbed $* \mathrm{OH}$, and removing the 9-layer Pt-Ni(111) slabs. The number of structures in the resulting training set is 201 . The final values of fitting parameters in Eq. [4] were $1.000 \times 10^{-8}, 9.414 \times 10^{-9}, 4.286$, and 2.986, respectively. The resulting cluster expansion had a RMS LOOCV error of $2.2 \mathrm{meV}$ per atom relative to DFT calculations. This cluster expansion is used to simulate the structural evolution (Ni dissolution and $\mathrm{Pt} / \mathrm{Ni}$ migration) during the experimental $\mathrm{CV}$ activation ${ }^{6,11}$ via kinetic Monte Carlo (KMC) as well as predict the thermodynamically stable Pt-Ni nanoparticles via Metropolis Monte Carlo. ${ }^{4,5}$ 


\section{Assessment of the accuracy of predicted $* \mathrm{OH}$ binding energies}

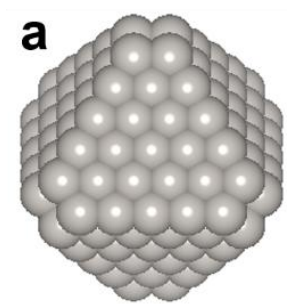

$1^{\text {st }}$ layer

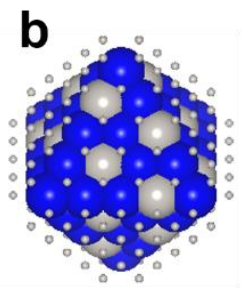

$2^{\text {nd }}$ layer
C

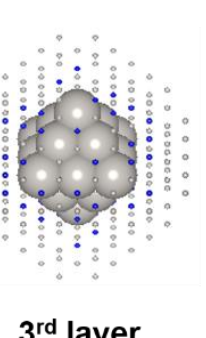

d<smiles></smiles>

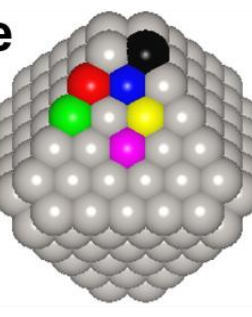

vertex edge-1 edge-2

face-1

face-2

face-3

Supplementary Figure 1. (a-d) The layer-by-layer atomic configuration of ground-state $\mathrm{Pt}_{178} \mathrm{Ni}_{47}$, which is predicted by the cluster expansion in vacuum (reported in our previous work $^{4,5}$ ). The 225-atom nanoparticle can be created by truncating one atom on each vertex of perfect octahedral 231-atom nanoparticle. (e) The six types of surface sites by their distinguished numbers of the nearest neighboring $\mathrm{Pt} / \mathrm{Ni}$ atoms and the $2^{\text {nd }}$-nearest neighboring atoms. 
Supplementary Table 1. The comparison of DFT-calculated and CE-predicted $* \mathrm{OH}$ binding energy on varied nanoparticles with a dilute ${ }^{*} \mathrm{OH}$ coverage. The surface sites on $\mathrm{Pt}_{178} \mathrm{Ni}_{47}$ were defined in Supplementary Figure 1e. and $\mathrm{Pt}_{225}$ was created by replacing all $\mathrm{Ni}$ atoms with $\mathrm{Pt}$ atoms. $\mathrm{Pt}_{79}, \mathrm{Pt}_{140}, \mathrm{Pt}_{225}, \mathrm{Pt}_{338}$, and $\mathrm{Pt}_{483}$ are octahedral nanoparticles with all vertex sites atoms truncated, where "surface-center" sites are the sites furthest to the edges. The generalized coordination number $(\mathrm{GCN})$ was firstly introduced by CalleVallejo et al. ${ }^{12,13}$

\begin{tabular}{|c|c|c|c|c|c|c|}
\hline particle & $\begin{array}{c}\text { surface } \\
\text { site }\end{array}$ & $\mathrm{CN}$ & $\mathrm{GCN}$ & $\begin{array}{c}\Delta \mathrm{E}_{\text {OH, }} \mathrm{DFT} \\
/ \mathrm{eV}\end{array}$ & $\begin{array}{c}\Delta \mathrm{E} \text { OH, CE } \\
/ \mathrm{eV}\end{array}$ & $\begin{array}{c}\text { diff } \\
/ \mathrm{eV}\end{array}$ \\
\hline $\mathrm{Pt}_{225}$ & face-1 & 9 & 7.5 & 1.082 & 0.979 & 0.103 \\
\hline & face-2 & 9 & 7.167 & 1.006 & 0.935 & 0.071 \\
\hline & face-3 & 9 & 6.667 & 0.992 & 0.902 & 0.090 \\
\hline & edge-1 & 7 & 5.167 & 0.660 & 0.640 & 0.020 \\
\hline & edge-2 & 7 & 5.083 & 0.653 & 0.614 & 0.039 \\
\hline $\mathrm{Pt}_{178} \mathrm{Ni}_{47}$ & vertex & 6 & 4.083 & 0.375 & 0.332 & 0.043 \\
\hline & face-1 & 9 & 7.5 & 1.322 & 1.323 & -0.001 \\
\hline & face-2 & 9 & 7.167 & 1.293 & 1.200 & 0.093 \\
\hline & edge-1 & 9 & 6.667 & 1.077 & 1.046 & 0.031 \\
\hline & edge-2 & 7 & 5.167 & 0.878 & 0.792 & 0.086 \\
\hline & vertex & 6 & 4.083 & 0.458 & 0.459 & -0.001 \\
\hline $\mathrm{Pt}_{79}$ & face-center & 9 & 7.5 & 0.955 & 0.891 & 0.064 \\
\hline $\mathrm{Pt}_{140}$ & face-center & 9 & 7.5 & 1.029 & 0.947 & 0.082 \\
\hline $\mathrm{Pt}_{225}$ & face-center & 9 & 7.5 & 1.082 & 0.979 & 0.103 \\
\hline $\mathrm{Pt}_{338}$ & face-center & 9 & 7.5 & 1.077 & 0.984 & 0.093 \\
\hline $\mathrm{Pt}_{483}$ & face-center & 9 & 7.5 & 1.115 & 0.994 & 0.121 \\
\hline
\end{tabular}




\section{Sabatier volcano relating $\Delta \mathrm{E}_{\mathrm{OH}}$ to activation free energy}

In this work, we assume the ORR occurs via the associative mechanism in acidic solution with an applied potential of $0.9 \mathrm{~V}$ (vs. the reversible hydrogen electrode, RHE) based on previous studies. ${ }^{8,14}$ The binding energies of three intermediate adsorbates $\left({ }^{*} \mathrm{OOH},{ }^{*} \mathrm{O}\right.$ and $\left.{ }^{*} \mathrm{OH}\right)$ are linearly related. ${ }^{15}$ The activation free energy along the reaction pathway $\left(\mathrm{E}_{a}\right)$ is correlated to oxygen binding energy $\left(\Delta \mathrm{E}_{\mathrm{O}}\right)$ through a Sabatier volcano plot. ${ }^{14,16,17}$ At an applied potential of $0.9 \mathrm{~V},{ }^{*} \mathrm{OH}$ is the most stable adsorbate on Pt-Ni surface. Thus we converted the Sabatier volcano as a function of oxygen binding energy to *OH binding energy by using the linear relationships among $* \mathrm{OOH},{ }^{*} \mathrm{O}$, and $* \mathrm{OH}^{15}$ Thus the activation free energy is expressed as a function of the ${ }^{*} \mathrm{OH}$ binding energy $\left(\Delta \mathrm{E}_{\mathrm{OH}}\right)$

$$
\mathrm{E}_{a}=-\min \left(-0.297+1.0\left(\Delta \mathrm{E}_{\mathrm{OH}}-1.153\right),-0.297+1.06\left(1.153-\Delta \mathrm{E}_{\mathrm{OH}}\right)\right) .
$$

where all energies are given in $\mathrm{eV}$ and peak position of Sabatier volcano is $\Delta \mathrm{E}_{\mathrm{OH}}$, peak $=1.115$ $\mathrm{eV}$. There is a difference between the peak position and $\mathrm{OH}$ binding energy on $\operatorname{Pt}(111)$ with a $1 / 4 \mathrm{ML}$ coverage $(\Delta \mathrm{E}$ OH, $\mathrm{Pt}(111)=1.035 \mathrm{eV})$, which is $\sim 0.1 \mathrm{eV}$ based on previous theoretical predictions ${ }^{8,18,19}$, and $\sim 0.135 \mathrm{eV}$ based on experimental measurements. ${ }^{20}$ The peak position $(\triangle \mathrm{E}$ OH, peak $=1.115 \mathrm{eV})$ was determined by adding the average of above theoretical and experimental differences, which is $(0.1+0.135) / 2 \approx 0.118 \mathrm{eV}$, to $\Delta \mathrm{E}_{\mathrm{OH}, \mathrm{Pt}(111)}$. 


\section{Kinetic Monte Carlo (KMC) simulation to predict ORR activities}

To estimate ORR specific activity, we have developed an approach in which the turnover frequency at each site is determined through a KMC simulation. The site-specific turnover frequency is expressed as

$$
\text { turnover frequency }=e^{-E_{a} / k T},
$$

where $\mathrm{E}_{a}$ is the activation free energy and calculated using the Sabatier volcano at varied $* \mathrm{OH}$ coverages.

This approach is similar to the one used in our previous work on $\mathrm{Pt}-\mathrm{Ni}(111)$ surfaces with one monolayer of adsorbed *O/Vacancy. ${ }^{17}$ We use a standard "rejection-free" KMC algorithm ${ }^{21,22}$ in which the only allowed transitions are adsorption and desorption. Each simulation was done on each independent nanoparticle, so the number of possible transition events for each KMC step is actually equal to the number of surface Pt atoms (i.e. 1596 for the intermetallic $\mathrm{Pt}_{3} \mathrm{Ni}$ nanoparticle in Fig. 1f). In this work, only surface atoms whose coordination number (nearest-neighboring $\mathrm{Pt} / \mathrm{Ni}$ atoms) is larger than 3 and smaller than 10 are considered as surface atoms. The transition rates (or turnover frequencies) for the transition events were calculated as $e^{\frac{-\mathrm{E}_{a}}{\mathrm{kT}}}$.

Specifically, for an adsorption event,

$$
\mathrm{E}_{a}=0.297+\max \left(0,1.06\left(\Delta \mathrm{E}_{\mathrm{OH}}-1.153\right)\right),
$$

where 1.115 is the peak position $\left(\Delta \mathrm{E}_{\mathrm{OH}}\right.$, peak $\left.=1.115 \mathrm{eV}\right)$ of the volcano plot. For a desorption event,

$$
\mathrm{E}_{a}=0.297+\max \left(0,1.0\left(1.153-\Delta \mathrm{E}_{\mathrm{OH}}\right)\right) .
$$

The maximum of $\mathrm{E}_{a}$ for an adsorption and a desorption event reproduces the equation of Sabatier volcano (Eq. [5]). After each event, the total elapsed "KMC time" was incremented by

$$
\Delta t_{K M C}=\left(\frac{1}{R_{N}}\right) * \ln \left(\frac{1}{u_{1}}\right)
$$


where $R_{N}$ is the total rate that sum all possible transition events, and $u_{1}$ is a uniform random number with $\mu_{1} \in(0,1]$. At the end of the run, the ORR specific activity $\left(S A_{\mathrm{KMC}}\right)$ was then calculated using

$$
S A_{\mathrm{KMC}}=S A_{0}\left(\frac{\mathrm{n} \text { (accepted desorption events })}{t_{\mathrm{KMC}}}\right)
$$

where $S A_{0}$ is a prefactor, $\mathrm{n}$ (accepted desorption events) is the number of accepted $* \mathrm{OH}$ desorption events, and $t_{\mathrm{KMC}}$ is the "KMC time". When $S A_{0}$ is the inverse of the number of adsorption sites on the surface of the studied nanoparticle (i.e. 1/1596 for the intermetallic $\mathrm{Pt}_{3} \mathrm{Ni}$ nanoparticle in Fig. 1f), then the KMC current agrees with the current calculated from the Sabatier volcano (Eq. [5]) in the limit of dilute coverage of *OH. The above specific activity $\left(S A_{\mathrm{KMC}}\right)$ can be converted to mass activity $\left(M A_{\mathrm{KMC}}\right)$ by multiplying the ratio of the number of surface Pt atoms $\left(N_{P t, \text { surface }}\right)$ to the number of total Pt atoms $\left(N_{P t}\right)$ for a particle.

$$
\begin{gathered}
\mathrm{S} A_{\mathrm{KMC}}=\frac{I}{N_{P t, \text { surface }}} \\
M A_{\mathrm{KMC}}=\frac{I}{N_{P t}}=\frac{\mathrm{S} A_{\mathrm{KMC}} \times N_{P t, \text { surface }}}{N_{P t}},
\end{gathered}
$$

where $I$ is the hypothetical total current.

To determine the atomic structures, 10 different octahedral $\mathrm{Pt}-\mathrm{Ni}$ nanoparticles with the same size and same Pt composition were randomly initialized, and 10 parallel KMC simulations of structural evolution (or Metropolis Monte Carlo simulations) were used to generate 10 different snapshots of activated disordered (or intermetallic) nanoparticles. To calculate the specific and mass activities, we ran a KMC simulation on each disordered or intermetallic nanoparticle. For each KMC simulation, the numbers of both equilibration steps and recording steps are 30 times the number of adsorption sites, and the predicted specific and mass activities and errors were average values and standard deviations (SA based on Eq. [10] and MA based on Eq. [12]) over 10 independent KMC runs on 10 nanoparticles. 


\section{Benchmark of ORR activities of commercial Pt/C}

The definitions of specific activity (SA) and mass activity (MA) for a single $\mathrm{Pt}-\mathrm{Ni}$ particle are expressed in Eq. [11] and Eq. [12]. To simulate the SA and MA of state-of-theart commercial Pt/C, we chose a cuboctahedron as the shape of pure Pt nanoparticles based on the Wulff construction of Pt demonstrated in the work of Li et al. ${ }^{23}$ The size (in diameter length) distribution of commercial Pt/C (Supplementary Table 2) was pulled from our previous work. ${ }^{6}$ The weighted average values of SA (3.04E-08) and MA (1.21E-08) for commercial $\mathrm{Pt} / \mathrm{C}$ were calculated according to the following expressions.

$$
\begin{gathered}
\langle S A\rangle=\frac{I}{N_{P t, \text { surface }}}=\frac{\sum_{i} I_{i} \times p_{i}}{\sum_{i} n_{P t, \text { surface }, i} \times p_{i}}=\frac{\sum_{i}\left(S A_{i} \times n_{P t, \text { sufface }, i}\right) \times p_{i}}{\sum_{i} n_{P t, \text { surface }, i} \times p_{i}} ; \\
\langle M A\rangle=\frac{I}{N_{P t}}=\frac{\sum_{i} I_{i} \times p_{i}}{\sum_{i} n_{P t, i} \times p_{i}}=\frac{\sum_{i}\left(S A_{i} \times n_{P t, \text { surface }, i}\right) \times p_{i}}{\sum_{i} n_{P t, i} \times p_{i}} .
\end{gathered}
$$

As shown in Supplementary Table 2, for each pure Pt particle with a specific diameter, $p_{i}$ is its percentage, and $n_{P t, \text { surface }, i}$ and $n_{P t, i}$ are the number of surface Pt atoms and the number of all $\mathrm{Pt}$ atoms. 
Supplementary Table 2. The simulated specific activity (SA) and mass activity (MA) of commercial $\mathrm{Pt} / \mathrm{C}$ with the size (in diameter) distribution of commercial $\mathrm{Pt} / \mathrm{C}$ pulled from our previous work. ${ }^{6}$ The percentage of particles listed for a specific diameter $\mathrm{d}$ is the total percentage of particles with the diameters in the range of $\mathrm{d} \pm 0.275 \mathrm{~nm}$. The nanoparticles with the diameters smaller than $2 \mathrm{~nm}$ have not been counted because the KMC-predicted ORR activities are likely to be less accurate at such small sizes due to quantum finite-size effects. The values of $S A_{i}$ were predicted using the KMC simulations.

\begin{tabular}{|c|c|c|c|c|c|c|}
\hline $\begin{array}{c}\text { cubo- } \\
\text { octahedron }\end{array}$ & $\begin{array}{c}\text { size in } \\
\text { diameter } \\
(\mathrm{nm})\end{array}$ & $\begin{array}{c}\text { percentage } \\
\left(p_{i}\right)\end{array}$ & $S A_{i}$ & $M A_{i}$ & $n_{P t, \text { sufface }, i}$ & $n_{P t, i}$ \\
\hline $\mathrm{Pt}_{5851}$ & 5.504 & 0 & $6.97 \mathrm{E}-8$ & $1.73 \mathrm{E}-8$ & 1452 & 5851 \\
\hline $\mathrm{Pt}_{4249}$ & 4.954 & 2.353 & $6.61 \mathrm{E}-8$ & $1.79 \mathrm{E}-8$ & 1148 & 4249 \\
\hline $\mathrm{Pt}_{3101}$ & 4.403 & 1.176 & $5.40 \mathrm{E}-8$ & $1.62 \mathrm{E}-8$ & 930 & 3101 \\
\hline $\mathrm{Pt}_{2075}$ & 3.853 & 5.882 & $4.78 \mathrm{E}-8$ & $1.59 \mathrm{E}-8$ & 690 & 2075 \\
\hline $\mathrm{Pt}_{1385}$ & 3.302 & 14.118 & $3.54 \mathrm{E}-8$ & $1.34 \mathrm{E}-8$ & 524 & 1385 \\
\hline $\mathrm{Pt}_{807}$ & 2.752 & 41.176 & $2.35 \mathrm{E}-8$ & $1.01 \mathrm{E}-8$ & 348 & 807 \\
\hline $\mathrm{Pt}_{459}$ & 2.202 & 35.295 & $1.45 \mathrm{E}-8$ & $7.39 \mathrm{E}-9$ & 234 & 459 \\
\hline $\begin{array}{c}\text { weighted } \\
\text { average of SA }\end{array}$ & & & $3.04 \mathrm{E}-8$ & & & \\
\hline $\begin{array}{c}\text { weighted } \\
\text { average of MA }\end{array}$ & & & & $1.21 \mathrm{E}-8$ & & \\
\hline
\end{tabular}

Using the Pt-Ni-OH@Pt-Vacancy cluster expansion, the predicted specific activity of $\mathrm{Pt}(111)$ on a $12 \times 12 \mathrm{Pt}(111)$ supercell is $1.15 \mathrm{E}-7$ (Supplementary Table 3), which is about 3.8 times that of commercial Pt/C (3.04E-8 in Supplementary Table 2) and agrees well with experiments $\left(5-10\right.$ times). ${ }^{24,25}$ The predicted specific activity of the theoretically identified ground state $\mathrm{Pt}_{29} \mathrm{Ni}_{7}(111){ }^{16}$ in equilibrium with bulk $\mathrm{Pt}_{3} \mathrm{Ni}$, which we will refer to as " $\mathrm{Pt}_{29} \mathrm{Ni}_{7}(111)$ ", is about 24.0 times that of commercial $\mathrm{Pt} / \mathrm{C}$ (3.04E-8). The $\mathrm{Pt}_{3} \mathrm{Ni}(111)$ catalyst (PNAS) 7-layer surface ${ }^{17}$ and $\mathrm{Pt}_{3} \mathrm{Ni}(111)$ catalyst (JPCC) 9-layer surface ${ }^{16}$ (Supplementary Table 3) were cluster-expansion-predicted surfaces closely matching the layer-by-layer $\mathrm{Pt}$ compositions of the highly active $\mathrm{Pt}_{3} \mathrm{Ni}(111)$ catalyst reported by Stamenkovic et al. ${ }^{26}$ 
Supplementary Table 3. The predicted specific activities of 7-layer and 9-layer Pt-Ni(111) surfaces compared with the predicted ones of 9-layer $\mathrm{Pt}(111)$ and commercial $\mathrm{Pt} / \mathrm{C}$ provided in Supplementary Table 2. The highly active $\mathrm{Pt}_{3} \mathrm{Ni}(111)$ surface reported by Stamenkovic et al. is also included. The specific activity simulations of 7-layer, and 9-layer $\mathrm{Pt}-\mathrm{Ni}(111)$ were conducted on a $12 \times 12$ (111) supercell with a vacuum thickness of 11-layer, 9-layer Pt/Ni atoms, respectively.

\begin{tabular}{|c|c|c|c|}
\hline prediction & $\mathrm{SA}_{\mathrm{KMC}}$ & $\begin{array}{l}\text { SA referenced to } \\
\text { commercial } \mathrm{Pt} / \mathrm{C}\end{array}$ & $\begin{array}{c}\text { SA referenced to } \\
\operatorname{Pt}(111)\end{array}$ \\
\hline $\operatorname{Pt}(111)$ & $1.15 \mathrm{E}-7$ & 3.8 & 1 \\
\hline $\mathrm{Pt}_{29} \mathrm{Ni}_{7}(111)$ & $7.28 \mathrm{E}-7$ & 24.0 & 6.3 \\
\hline $\begin{array}{c}\mathrm{Pt}_{3} \mathrm{Ni}(111) \text { catalyst } \\
\text { (PNAS) }\end{array}$ & $6.18 \mathrm{E}-07$ & 20.3 & 5.4 \\
\hline $\begin{array}{c}\mathrm{Pt}_{3} \mathrm{Ni}(111) \text { catalyst } \\
\text { (JPCC) }\end{array}$ & $7.10 \mathrm{E}-07$ & 23.4 & 6.2 \\
\hline $\mathrm{Pt}_{25} \mathrm{Ni}_{11}(111)-\mathrm{a}$ & $1.22 \mathrm{E}-07$ & 4.0 & 1.1 \\
\hline $\mathrm{Pt}_{25} \mathrm{Ni}_{11}(111)-\mathrm{b}$ & $9.14 \mathrm{E}-10$ & 0.03 & 0.01 \\
\hline experiment & $\mathrm{SA}\left(\mathrm{mA} / \mathrm{cm}^{2}\right)$ & $\begin{array}{l}\text { SA referenced to } \\
\text { commercial } \mathrm{Pt} / \mathrm{C}\end{array}$ & $\begin{array}{c}\text { SA referenced to } \\
\operatorname{Pt}(111)\end{array}$ \\
\hline $\begin{array}{c}\mathrm{Pt}_{3} \mathrm{Ni}(111) \text { reported } \\
\text { by Stamenkovic at } \\
\text { al. }^{26}\end{array}$ & 18 & 90 & 10 \\
\hline
\end{tabular}

Note: $\mathrm{Pt}_{25} \mathrm{Ni}_{11}(111)$-a and $\mathrm{Pt}_{25} \mathrm{Ni}_{11}(111)$-b are 9-layer surfaces with a Pt-skin in the $1^{\text {st }}$ layer and ordered $\mathrm{Pt}_{3} \mathrm{Ni}$ in the $4^{\text {th }}$ and $5^{\text {th }}$ layers. ${ }^{16}$ The $2^{\text {nd }}$ and $3^{\text {rd }}$ layers are $75 \%$ and $25 \% \mathrm{Pt}$ for $\mathrm{Pt}_{25} \mathrm{Ni}_{11}(111)$-a, and $100 \%$ and zero $\mathrm{Pt}$ for $\mathrm{Pt}_{25} \mathrm{Ni}_{11}(111)$-b. 

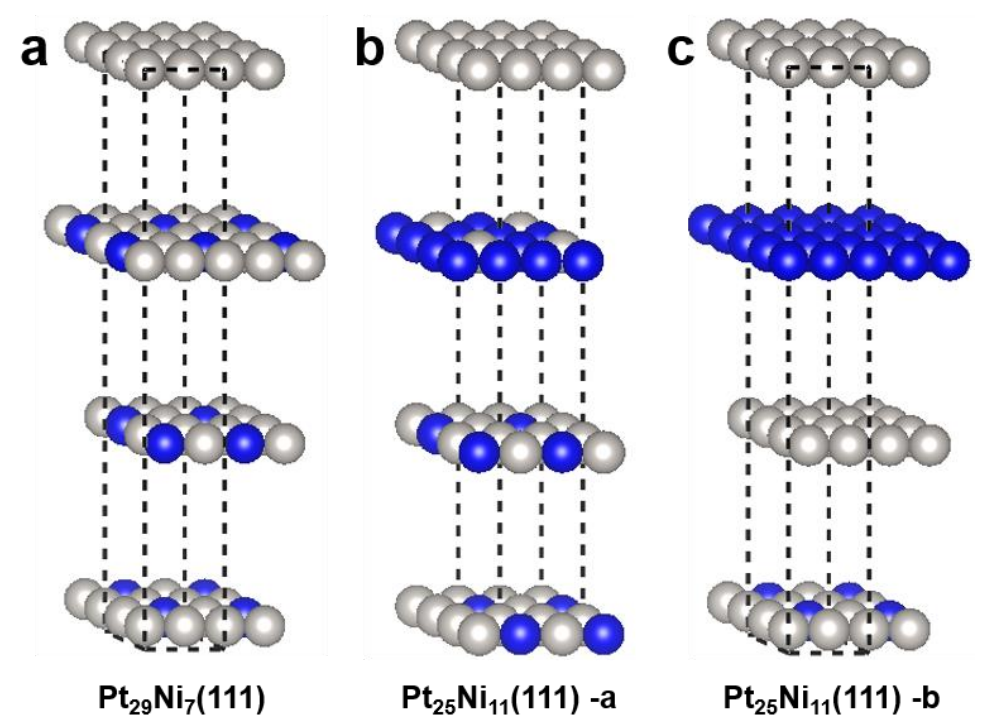

Supplementary Figure 2. The top four layers of three symmetric 9-layer surfaces with a lattice parameter of intermetallic bulk $\mathrm{Pt}_{3} \mathrm{Ni}$. (a, c) ground state surfaces in equilibrium with bulk $\mathrm{Pt}_{3} \mathrm{Ni}$ at $0 \mathrm{~K}$ predicted in our previous work ${ }^{16}$; (b) a dummy surface with $25 \%$, $75 \%$, and $75 \% \mathrm{Pt}$ in the $2^{\text {nd }}, 3^{\text {rd }}, 4^{\text {th }}$ layers, respectively. The grey and blue spheres are $\mathrm{Pt}$ and $\mathrm{Ni}$ atoms respectively.

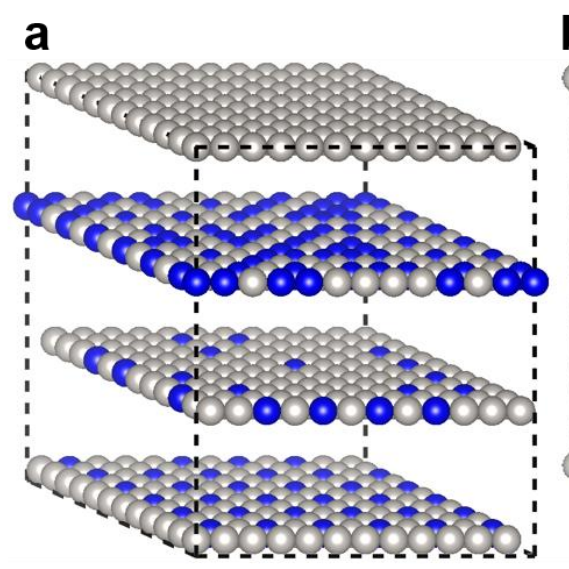

closely matching record-setting catalyst (PNAS)

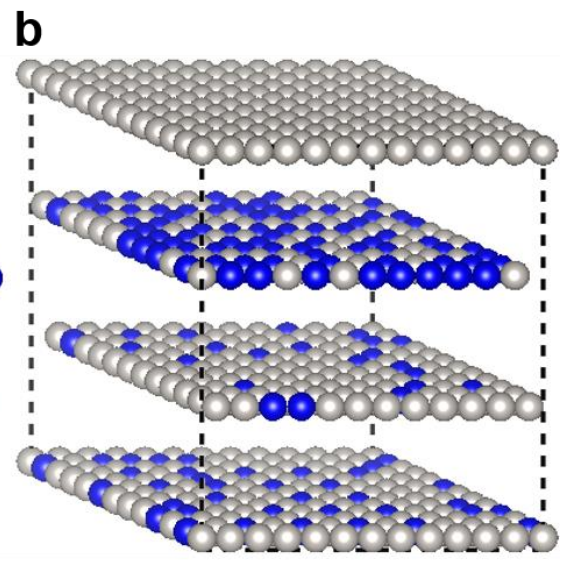

closely matching record-setting catalyst (JPCC)

Supplementary Figure 3. The top four layers of two snapshot slabs with a $12 \times 12$ (111) unit cell from Monte Carlo simulations. (a) a 7-layer snapshot ${ }^{17}$ and (c) a 9-layer snapshot ${ }^{16}$ matching the layer-by-layer $\mathrm{Pt}$ compositions of the highly active $\mathrm{Pt}_{3} \mathrm{Ni}(111)$ catalyst reported by Stamenkovic et al. ${ }^{26}$ The grey and blue spheres are $\mathrm{Pt}$ and $\mathrm{Ni}$ atoms respectively. 


\section{Predicted Ni loss of activated disordered Pt-Ni nanoparticles}
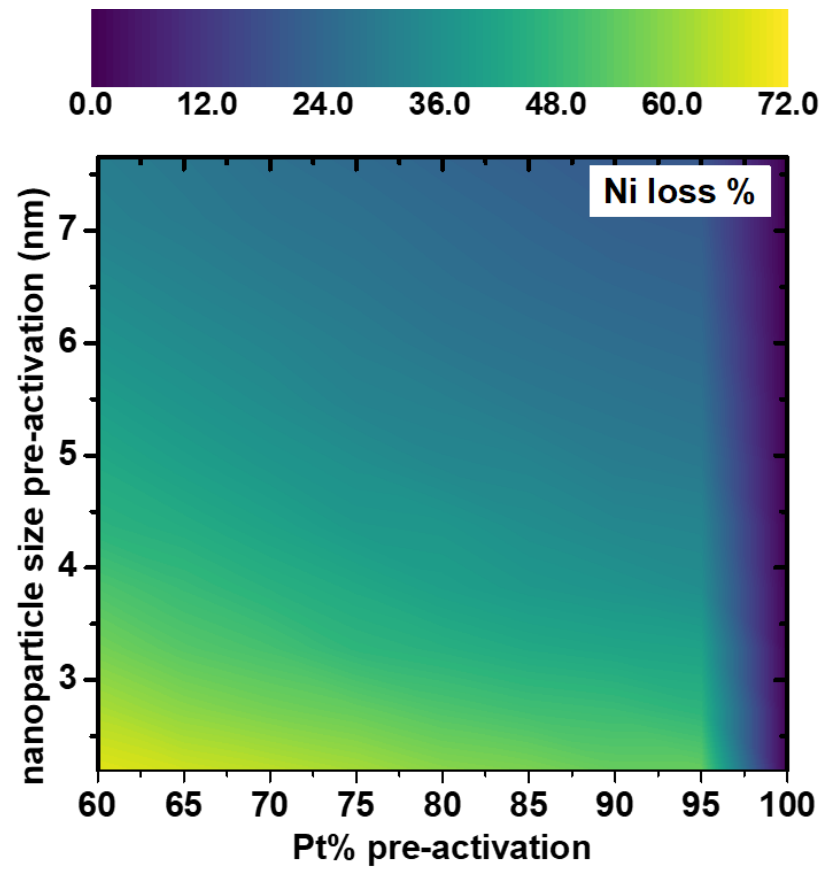

Supplementary Figure 4. Predicted Ni loss of activated disordered Pt-Ni nanoparticles. The $\mathrm{x}$-axis is the Pt composition after KMC with an increment of 5\%, and the $\mathrm{y}$-axis is the edge length before $\mathrm{KMC}$ (edge length). All specific and mass activity values are referenced to those of simulated commercial Pt/C. 
7. Precited specific and mass activities of $3.3 \mathbf{~ n m}$ disordered particles
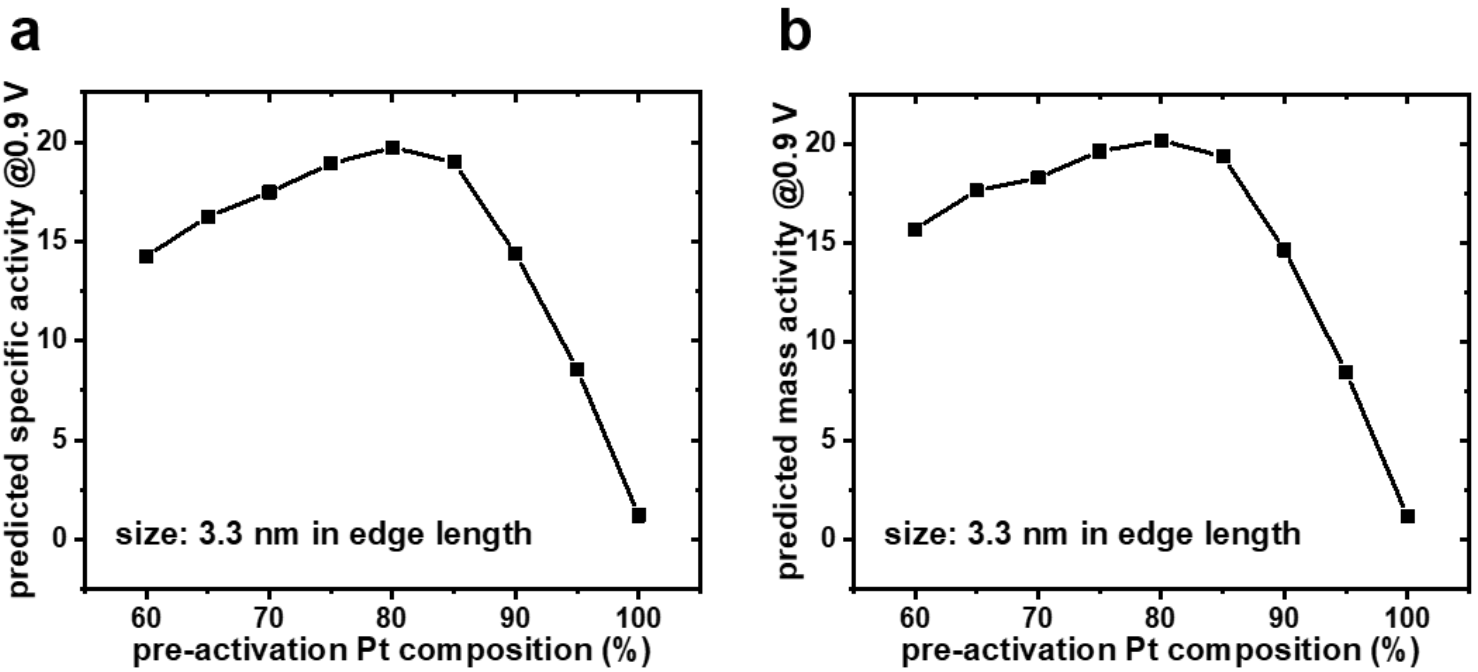

Supplementary Figure 5. The predicted (a) specific activity and (b) mass activity of 3.3 $\mathrm{nm}$ activated disorder $\mathrm{Pt}-\mathrm{Ni}$ particles as a function of pre-activation Pt composition. The values of activities are referenced to those of commercial $\mathrm{Pt} / \mathrm{C}$. 


\section{Predicted average *OH binding energies of post-activated disordered 6175-atom nanoparticles as a function of Pt composition}

a

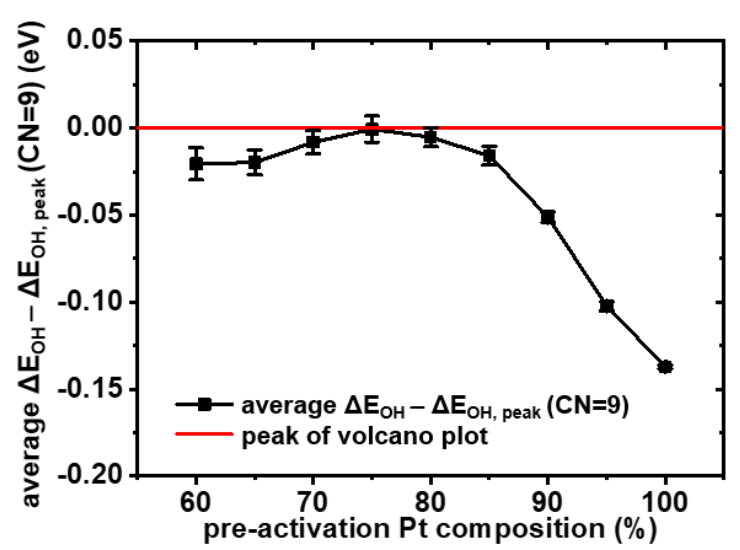

b

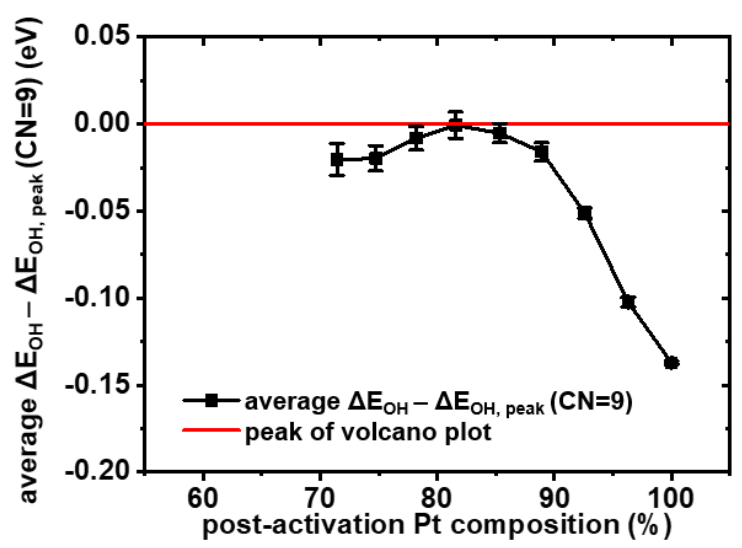

Supplementary Figure 6. The predicted average $* \mathrm{OH}$ binding energies, referenced to that of volcano plot peak, on surface sites with coordination number equal to 9 for activated disordered octahedral nanoparticles with initial 6175 atoms as a function of $\mathrm{Pt} \%$ (a) before $\mathrm{KMC}$ (pre-activation) and (b) after KMC (post-activation). 
9. Catalytic activity maps as a function of post-activated Pt composition and preactivated particle size
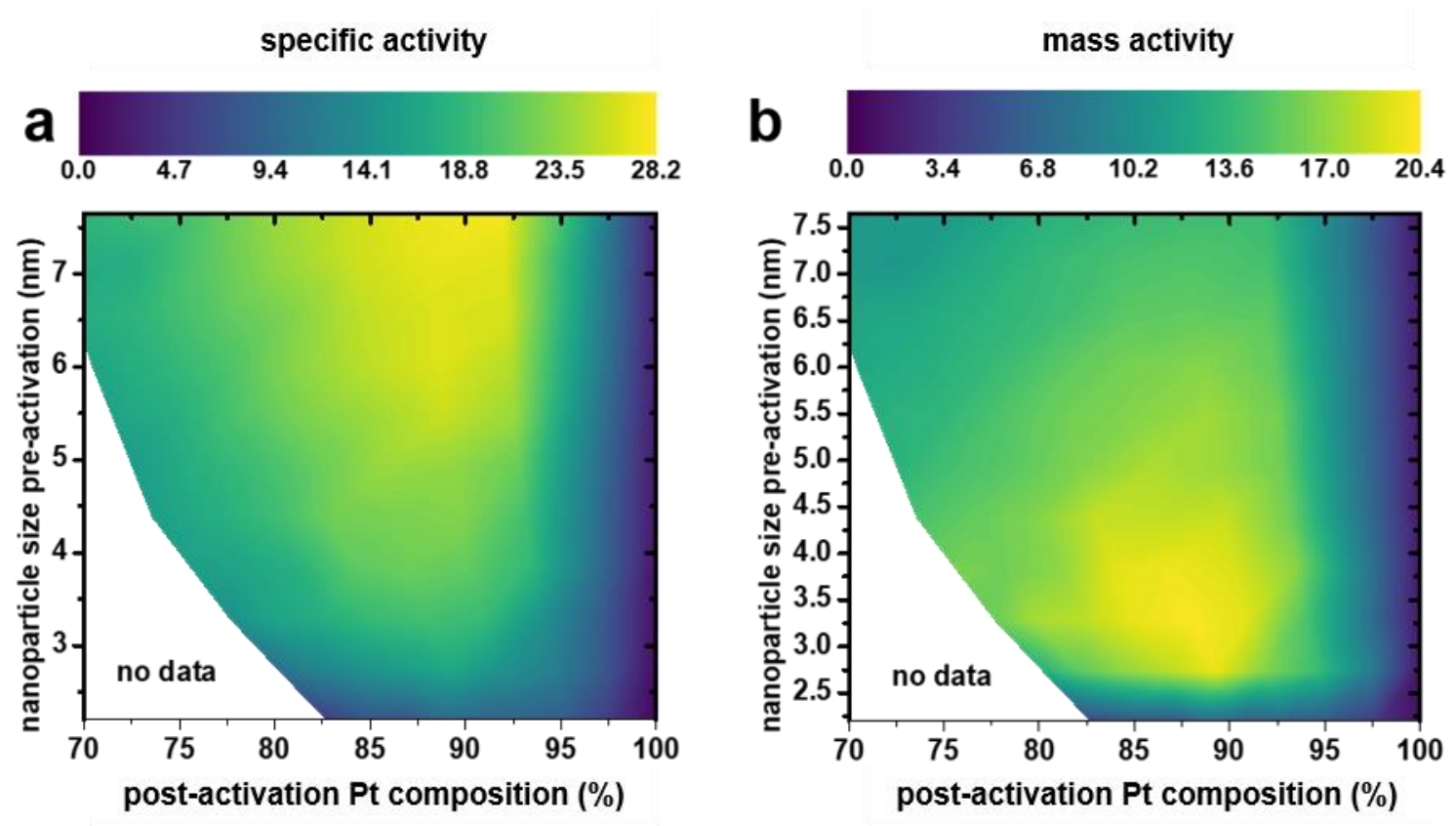

Supplementary Figure 7. Predicted size-composition catalytic activity maps for the ORR. (a) Specific activity and (b) mass activity of activated disordered Pt-Ni nanoparticles. The $\mathrm{x}$-axis is the Pt composition after KMC (post-activation), and the $\mathrm{y}$-axis is the edge length before KMC (pre-activation). All specific and mass activity values are referenced to those of simulated commercial $\mathrm{Pt} / \mathrm{C}$. 


\section{Vacancy formation energies and sub-surface Pt vacancies}

Supplementary Table 4. On the (111) surfaces of 225-atom octahedral nanoparticles and $\mathrm{Pt}(111)$ as well as representative Pt-rich (111) surfaces, the DFT-calculated vacancy formation energy (VFE) on the $1^{\text {st }}$ and $2^{\text {nd }}$ layers.

\begin{tabular}{|c|c|c|c|}
\hline structure & $\begin{array}{l}\text { position of } \mathrm{Pt} \\
\text { vacancy }\end{array}$ & $\begin{array}{l}\text { vacancy formation } \\
\text { energy (VFE) / eV }\end{array}$ & $\begin{array}{l}\text { difference of VFE } \\
\text { between the } 2^{\text {nd }} \text { and } \\
1^{\text {st }} \text { layers } / \mathrm{eV}\end{array}$ \\
\hline \multirow[t]{2}{*}{$\mathrm{Pt}_{225}$ particle } & $1^{\text {st }}$ layer & 0.990 & \multirow[b]{2}{*}{-1.070} \\
\hline & $2^{\text {nd }}$ layer & -0.080 & \\
\hline \multirow[t]{2}{*}{$\mathrm{Pt}_{206} \mathrm{Ni}_{19}$ particle } & $1^{\text {st }}$ layer & 1.260 & \multirow[b]{2}{*}{-0.867} \\
\hline & $2^{\text {nd }}$ layer & 0.393 & \\
\hline \multirow{2}{*}{$\begin{array}{l}\operatorname{Pt}(111) \text { with } 2 \times 2 \\
\text { supercell }\end{array}$} & $1^{\text {st }}$ layer & 0.895 & \multirow[b]{2}{*}{-0.710} \\
\hline & $2^{\text {nd }}$ layer & 0.184 & \\
\hline \multirow{2}{*}{$\begin{array}{l}\operatorname{Pt}(111) \text { with } 4 \times 4 \\
\text { supercell }\end{array}$} & $1^{\text {st }}$ layer & 1.081 & \multirow[b]{2}{*}{-0.653} \\
\hline & $2^{\text {nd }}$ layer & 0.428 & \\
\hline \multirow{2}{*}{$\begin{array}{l}\mathrm{Pt}_{29} \mathrm{Ni}_{7}(111) \quad \text { with } \\
2 \times 2 \text { supercell }\end{array}$} & $1^{\text {st }}$ layer & 1.209 & \multirow[b]{2}{*}{-0.562} \\
\hline & $2^{\text {nd }}$ layer & 0.67 & \\
\hline \multirow{2}{*}{$\begin{array}{l}\mathrm{Pt}_{29} \mathrm{Ni}_{7}(111) \quad \text { with } \\
4 \times 4 \text { supercell }\end{array}$} & $1^{\text {st }}$ layer & 1.291 & \multirow[b]{2}{*}{-0.578} \\
\hline & $2^{\text {nd }}$ layer & 0.712 & \\
\hline
\end{tabular}

Note: $\mathrm{Pt}_{225}$ particle is the octahedral particle with six vertex-site $\mathrm{Pt}$ atoms removed (the shape is the same as the $\mathrm{Pt}_{178} \mathrm{Ni}_{47}$ particle in Supplementary Figure 1; $\mathrm{Pt}_{206} \mathrm{Ni}_{19}$ particle is the $\mathrm{Pt}_{225}$ particle with $\mathrm{Pt}$ atoms deeper than the $2^{\text {nd }}$ layer replaced by $\mathrm{Ni}$ atoms; $\mathrm{Pt}_{29} \mathrm{Ni}_{7}(111)$ is the surface illustrated in Supplementary Figure 2a. 

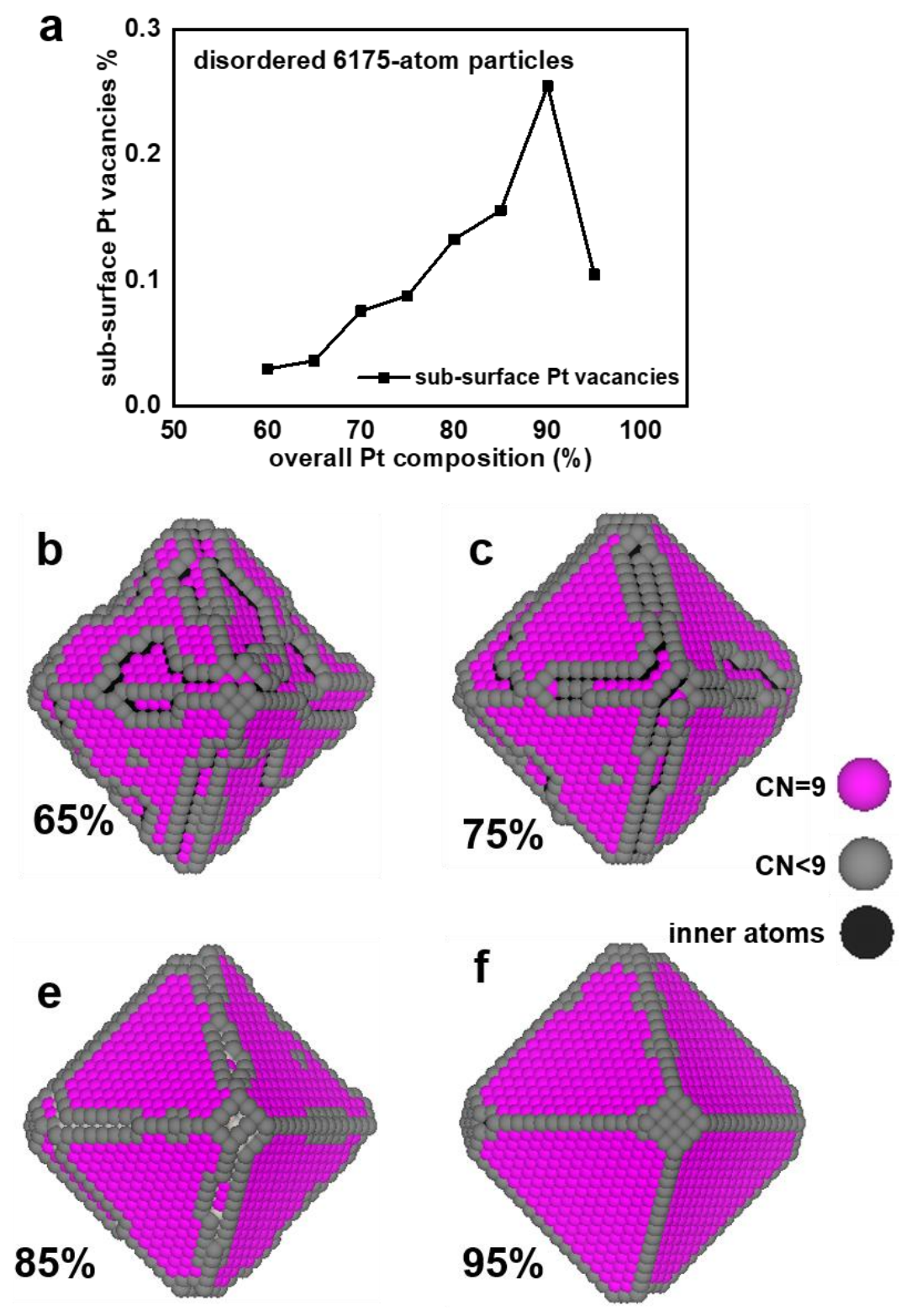

Supplementary Figure 8. The (a) concentration of sub-surface Pt vacancies of activated disordered Pt-Ni particles as a function of the pre-activated Pt composition (b-e) Distribution of surface site coordination numbers of activated disordered particles with preactivated Pt composition of (b) $65 \%$, (c) $75 \%$, (d) $85 \%$, (e) 95\%. In figure b-e, the surface sites on (111) surface with coordination numbers less than 9 have nearest-neighboring subsurface Pt vacancies. 


\section{Determination of atomic structures of activated disordered Pt-Ni particles with Pt-rich edges}

According to the experimental observation of Pt-rich edges for octahedral $\mathrm{Pt}^{-\mathrm{Ni}}$ nanoparticles from Strasser et al.'s work ${ }^{27}$, we assume that the deposition rate of Pt atoms on edge sites is faster than that on (111) facet sites, and define the degree to which edges are more Pt-rich than (111) facets as $A$ using the following equation,

$$
A=\frac{\frac{r_{P t}(e)}{r_{N i}(e)}}{\frac{r_{P t}(f)}{r_{N i}(f)}}=\frac{f_{P t, e}}{f_{N i, e}} / \frac{r_{P t, f}}{r_{N i, f}},
$$

where the related notations are defined as follows:

$\frac{r_{P t}(e)}{r_{N i}(e)}$ is the deposition rate of Pt referenced to that of Ni on edge sites;

$\frac{r_{P t}(f)}{r_{N i}(f)}$ is the deposition rate of Pt referenced to Ni on facet sites;

$f_{P t, e}$ is the fraction of Pt on the edge sites;

$f_{N i, e}$ is the fraction of $\mathrm{Ni}$ on the edge sites and is equivalent to $\left(1-f_{P t, e}\right)$;

$f_{P t, f}$ is the fraction of Pt on the face sites;

$f_{N i, f}$ is the fraction of $\mathrm{Ni}$ on the face sites and is equivalent to $\left(1-f_{P t, f}\right)$.

Then $f_{N i, f}$ can be calculated using the following equation:

$$
f_{N i, f}=\frac{2 A f_{N i}}{\left[f_{e}+A f_{f}+(A-1) f_{N i}\right]+\sqrt{\left[f_{e}+A f_{f}+(A-1) f_{N i}\right]^{2}+4 A f_{N i}(1-A) f_{f}}},
$$

where the related definitions are as follows.

$f_{e}$ is the fraction of total atoms on the edge sites; 
$f_{f}$ is the fraction of total atoms on the face sites and is equivalent to $\left(1-f_{e}\right)$;

$f_{N i}$ is the total fraction of $\mathrm{Ni}$ atoms within the nanoparticles;

$f_{P t}$ is the total fraction of $\mathrm{Pt}$ atoms within the nanoparticles and is equivalent to $\left(1-f_{N i}\right)$;

$f_{N i, e}$ is the fraction of $\mathrm{Ni}$ on the edge sites and is equivalent to $\left(f_{N i}-f_{f} * f_{N i, f}\right) / f_{e}$.

For a 6175-atom octahedral nanoparticle, we define the width of each edge as the threelayers of atoms along the edge and immediately adjacent to the edge, which means there are 3199 atoms on edge sites and 2976 atoms on face sites $\left(f_{e}=3199 / 6175\right.$ and $f_{f}=1-f_{e}$ ). The $f_{N i, f}$ and corresponding distributions of $\mathrm{Pt}$ and $\mathrm{Ni}$ atoms within the whole Pt-Ni nanoparticles with Pt-rich edges are demonstrated in Supplementary Table 5 for $\mathrm{A}=5$. Accordingly the $\mathrm{Pt}$ and $\mathrm{Ni}$ atoms are randomly distributed to initialize the disordered particles, then atomic structures of activated disordered particles are simulated using the kinetic Monte Carlo simulations. The predicted average specific activities over 10 independent snapshot structures for each individual overall $\mathrm{Pt}$ composition are demonstrated for $A=3,5,10$, and $+\infty$ in Supplementary Figure 9. We observed little change in the composition (about $85 \% \mathrm{Pt}$ for pre-activated particles) predicted to maximize activity across all values of $A$. 
Supplementary Table 5. The distributions of Pt and Ni atoms between edge and face sites across the octahedral Pt-Ni nanoparticles with Pt-rich edges for $A=5$.

\begin{tabular}{|c|c|c|c|c|c|c|}
\hline \multirow{2}{*}{$f_{P t}$} & \multirow{2}{*}{$f_{N i, f}$} & $f_{N i, e}$ & \multicolumn{4}{|c|}{ distributions of Pt and Ni atoms } \\
\cline { 4 - 7 } & & & $N_{N i, e}$ & $N_{P_{t, e}}$ & $N_{N i, f}$ & $N_{P_{t, f}}$ \\
\hline 0.6 & 0.5899 & 0.2234 & 715 & 2484 & 1755 & 1221 \\
\hline 0.65 & 0.5290 & 0.1834 & 587 & 2612 & 1574 & 1402 \\
\hline 0.7 & 0.4639 & 0.1475 & 472 & 2727 & 1381 & 1595 \\
\hline 0.75 & 0.3947 & 0.1154 & 369 & 2830 & 1175 & 1801 \\
\hline 0.8 & 0.3218 & 0.0867 & 277 & 2922 & 958 & 2018 \\
\hline 0.85 & 0.2455 & 0.0611 & 196 & 3003 & 731 & 2245 \\
\hline 0.9 & 0.1663 & 0.0384 & 123 & 3076 & 495 & 2481 \\
\hline 0.95 & 0.0843 & 0.0180 & 58 & 3141 & 251 & 2725 \\
\hline
\end{tabular}
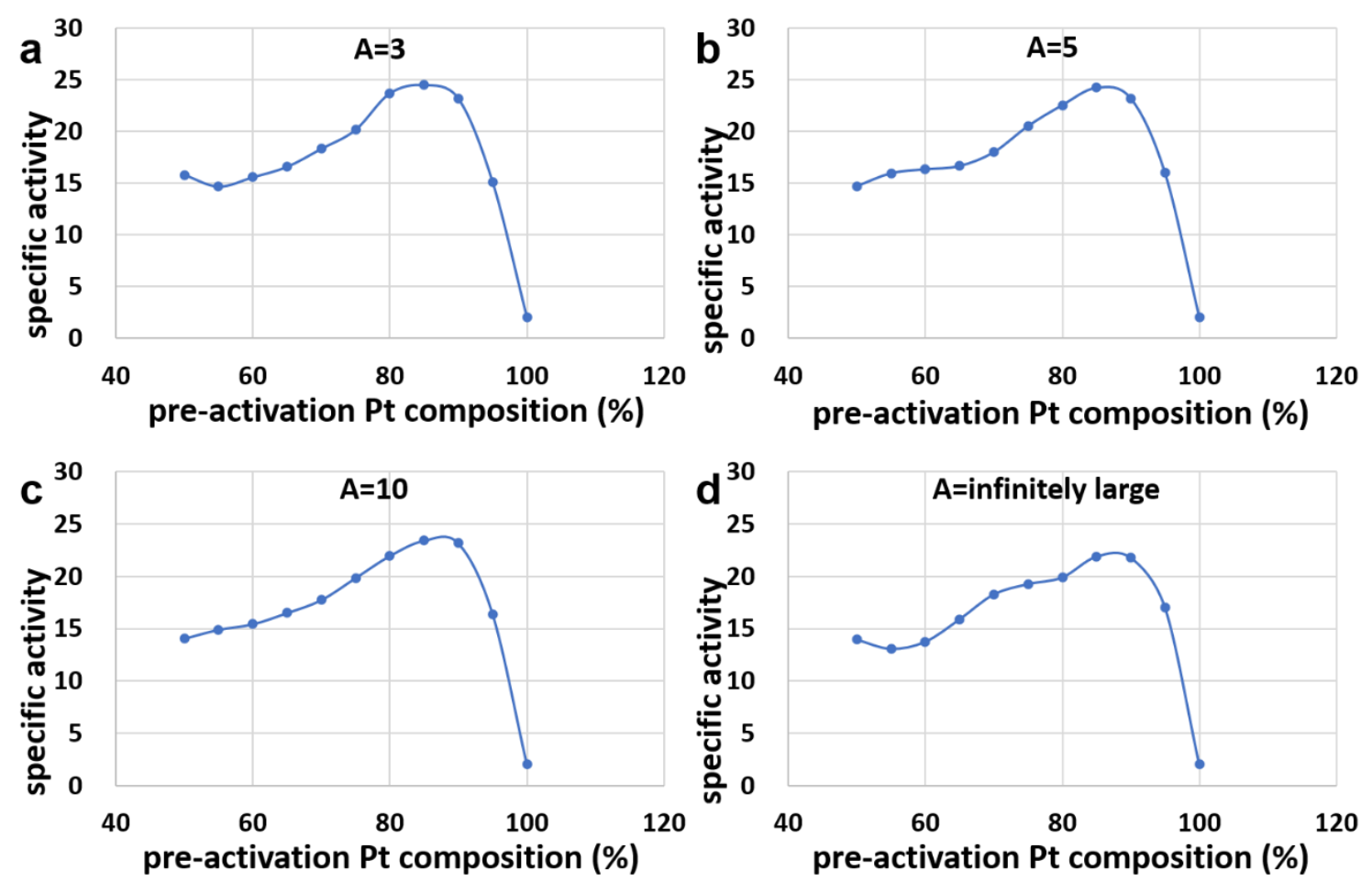

Supplementary Figure 9. The average specific activities (over 10 independent snapshot structures) of activated $\mathrm{Pt}-\mathrm{Ni}$ particles with Pt-rich edges as a function of pre-activation $\mathrm{Pt}$ composition for (a) $\mathrm{A}=3$, (b) $\mathrm{A}=5$, (c) $\mathrm{A}=10$, and (d) $\mathrm{A}=$ infinitely large, respectively. 


\section{Layer-by-layer atomic structures of disordered $\mathrm{Pt}_{0.85 \mathrm{Ni}} \mathrm{N}_{0.15}$ nanoparticles}

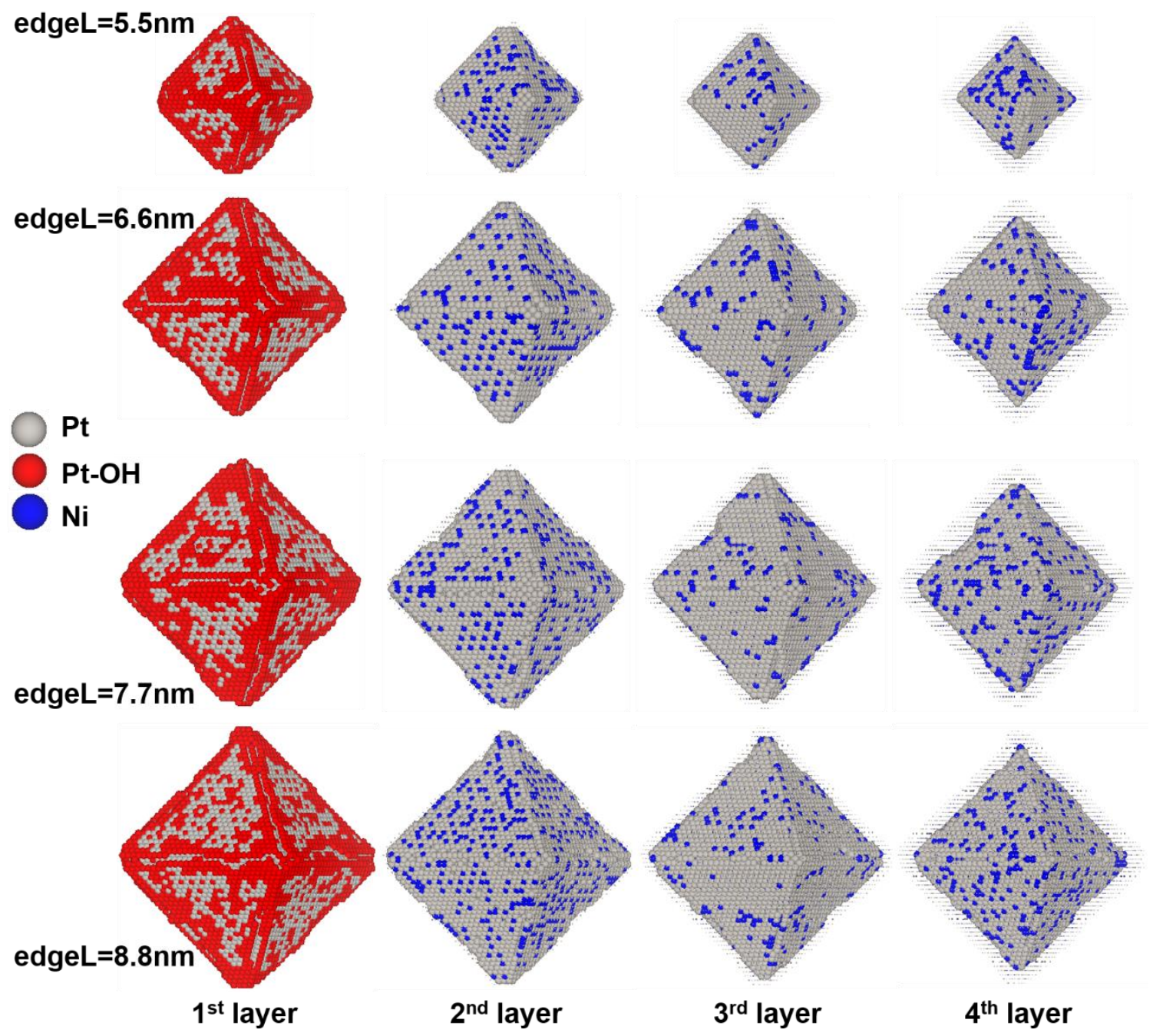

Supplementary Figure 10. The layer-by-layer atomic structures of representative snapshots at $298 \mathrm{~K}$ for disordered $\mathrm{Pt}_{0.85} \mathrm{Ni}_{0.15}$ nanoparticles with adsorbed $* \mathrm{OH}$ determined by a $\mathrm{KMC}$ run on nanoparticles with edge lengths (before activation) of $5.5 \mathrm{~nm}, 6.6 \mathrm{~nm}$, $7.7 \mathrm{~nm}$, and $8.8 \mathrm{~nm}$. 

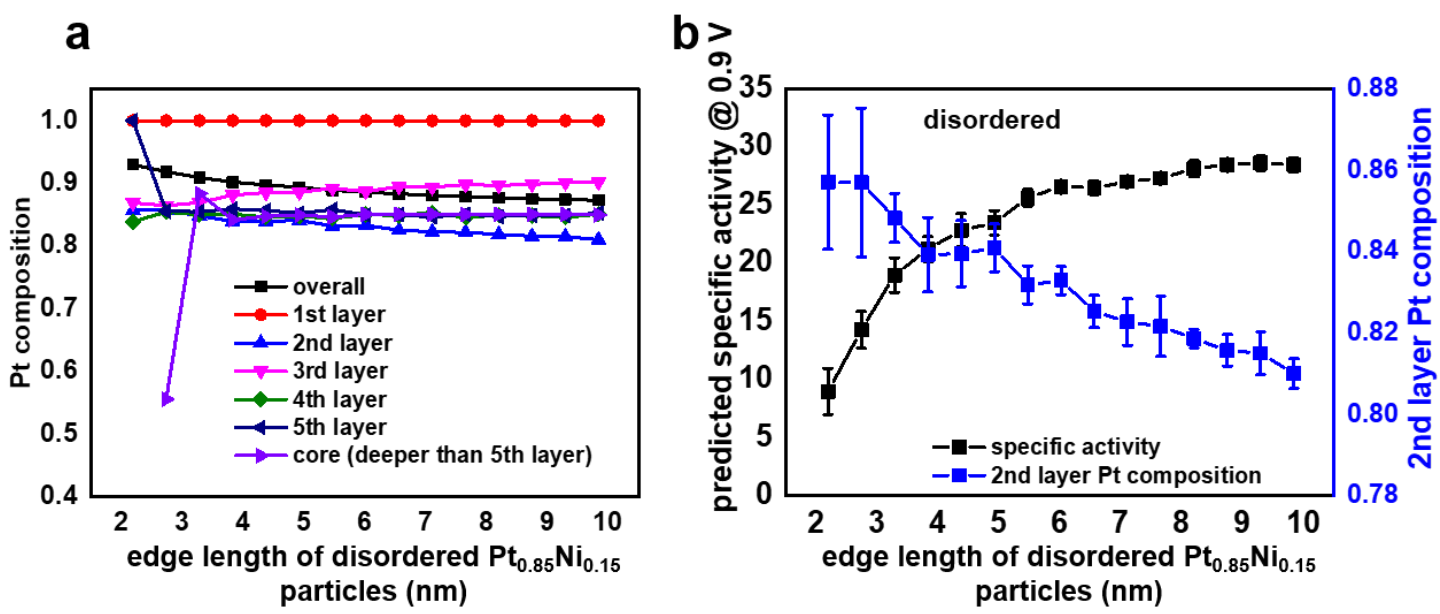

Supplementary Figure 11. The (a) layer-by-layer Pt composition and (b) predicted specific activity compared with the $2^{\text {nd }}$ layer $\mathrm{Pt}$ composition for disordered $\mathrm{Pt}_{0.85} \mathrm{Ni}_{0.15}$ nanoparticles as a function of particle edge length. 
Supplementary Table 6. The predicted layer-by-layer Pt composition for disordered $\mathrm{Pt}_{0.85} \mathrm{Ni}_{0.15}$ nanoparticles at $298 \mathrm{~K}$ as a function of edge length from $2.2 \mathrm{~nm}$ to $9.9 \mathrm{~nm}$.

\begin{tabular}{|c|c|c|c|c|c|c|}
\hline \multirow{2}{*}{$\begin{array}{c}\text { size in } \\
\text { edge } \\
\text { ength } \\
(\mathrm{nm})\end{array}$} & \multicolumn{6}{|c|}{ Pt composition (\%) } \\
\cline { 2 - 7 } & overall & $1^{\text {st }}$ layer & $2^{\text {nd }}$ layer & $3^{\text {rd }}$ layer & $4^{\text {th }}$ layer & $5^{\text {th }}$ layer \\
\hline 2.19 & 93.0 & 100 & 85.7 & 86.9 & 83.9 & 100 \\
\hline 2.73 & 91.9 & 100 & 85.7 & 86.4 & 85.4 & 85.6 \\
\hline 3.28 & 90.9 & 100 & 84.8 & 86.9 & 85.0 & 85.5 \\
\hline 3.83 & 90.2 & 100 & 83.9 & 88.1 & 85.1 & 85.9 \\
\hline 4.37 & 89.7 & 100 & 84.0 & 88.5 & 84.6 & 85.7 \\
\hline 4.92 & 89.4 & 100 & 84.1 & 88.6 & 84.7 & 85.2 \\
\hline 5.47 & 88.9 & 100 & 83.2 & 89.2 & 84.4 & 85.8 \\
\hline 6.01 & 88.6 & 100 & 83.3 & 88.7 & 85.0 & 85.0 \\
\hline 6.56 & 88.3 & 100 & 82.5 & 89.5 & 84.9 & 85.0 \\
\hline 7.11 & 88.1 & 100 & 82.3 & 89.4 & 85.3 & 84.6 \\
\hline 7.66 & 87.9 & 100 & 82.2 & 89.8 & 84.6 & 85.1 \\
\hline 8.20 & 87.7 & 100 & 81.9 & 89.7 & 84.9 & 84.7 \\
\hline 8.75 & 87.5 & 100 & 81.6 & 89.8 & 84.8 & 84.9 \\
\hline 9.30 & 87.4 & 100 & 81.5 & 90.1 & 84.6 & 84.9 \\
\hline 9.84 & 87.3 & 100 & 81.0 & 90.2 & 85.1 & 85.2 \\
\hline
\end{tabular}


13. Average $* \mathrm{OH}$ binding energies on (111) sites with $\mathrm{CN}=9$ for disordered $\mathrm{Pt}_{0.85} \mathrm{Ni}_{0.15}$ nanoparticles

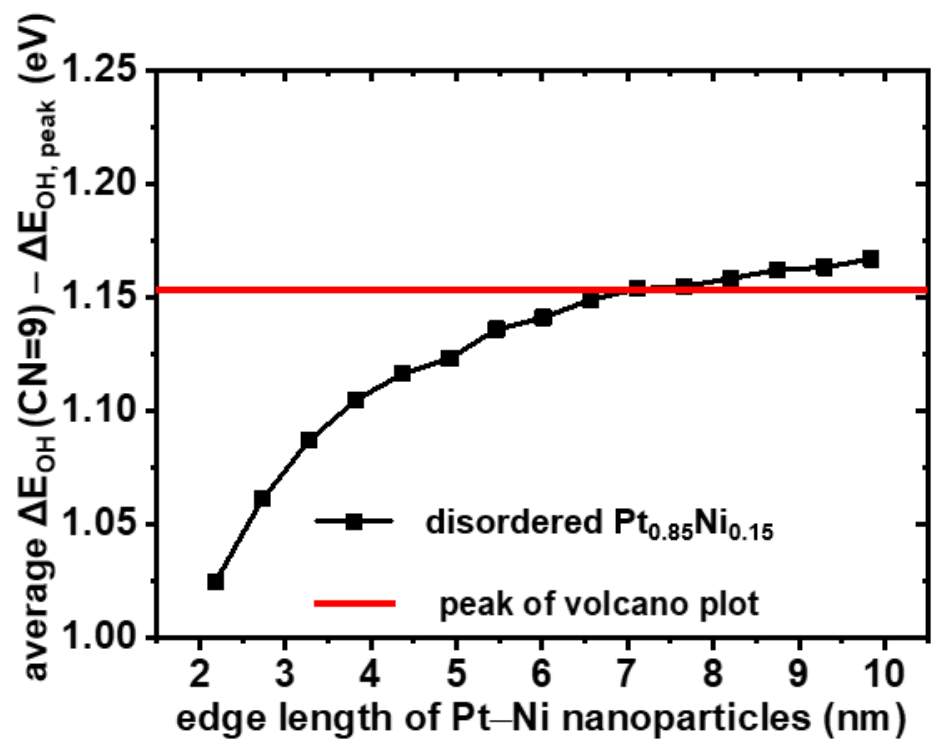

Supplementary Figure 12. Average $* \mathrm{OH}$ binding energies on (111) sites with $\mathrm{CN}=9$ for disordered $\mathrm{Pt}_{0.85} \mathrm{Ni}_{0.15}$ nanoparticles a function of particle size (edge length). The horizontal red line indicates the volcano plot peak. 


\section{Determination of chemical potentials of $\mathrm{Pt}$ and Ni maximizing catalytic activities of intermetallic Pt-Ni particles}

To determine the atomic structures of intermetallic $\mathrm{Pt}_{3} \mathrm{Ni}$ particles with maximal specific and mass activities, we have run Metropolis Monte Carlo ${ }^{28}$ simulations under a grand canonical ensemble within the chemical potential window where bulk ordered $\mathrm{Pt}_{3} \mathrm{Ni}$ is stable. To perform above simulations, firstly we refer the chemical potential difference between $\mathrm{Pt}$ and $\mathrm{Ni}$ as $\mu \equiv \mu(\mathrm{Pt})-\mu(\mathrm{Ni})$, where reference chemical potentials of the bulk metals are set to zero. Using the same strategy in our previous work ${ }^{16,17}$, the window of $\mu$ where bulk $\mathrm{Pt}_{3} \mathrm{Ni}$ is stable is between $0.04 \mathrm{eV}$ and $0.50 \mathrm{eV}$ according to the energies of bulk $\mathrm{Pt}$, ordered $\mathrm{Pt}_{3} \mathrm{Ni}$ and ordered $\mathrm{Pt}_{2} \mathrm{Ni}_{2}$ predicted by $\mathrm{Pt}-\mathrm{Ni}-\mathrm{Vacancy}$ cluster expansion in section 1.2. Within the $\mu$ window between 0.04 and $0.50 \mathrm{eV}$ (with a grid of $0.025 \mathrm{eV}$ ), the thermodynamically stable atomic structures of 6175-atom nanoparticles are simulated using the Metropolis Monte Carlo simulations. The corresponding averaged specific and mass activities over 10 thermodynamic snapshot structures for each chosen value of $\mu$ are shown as a function of $\mu$ in Supplementary Figure 13b. The maximal activities are achieved at $\mu=0.45 \mathrm{eV}$ and an overall Pt composition of $\sim 82 \%$. Thus in the present work we will choose $\mu=0.45 \mathrm{eV}$ when running Metropolis Monte Carlo simulations to determine the atomic structures of intermetallic $\mathrm{Pt}-\mathrm{Ni}$ octahedral particles with maximal activities at varied sizes. 

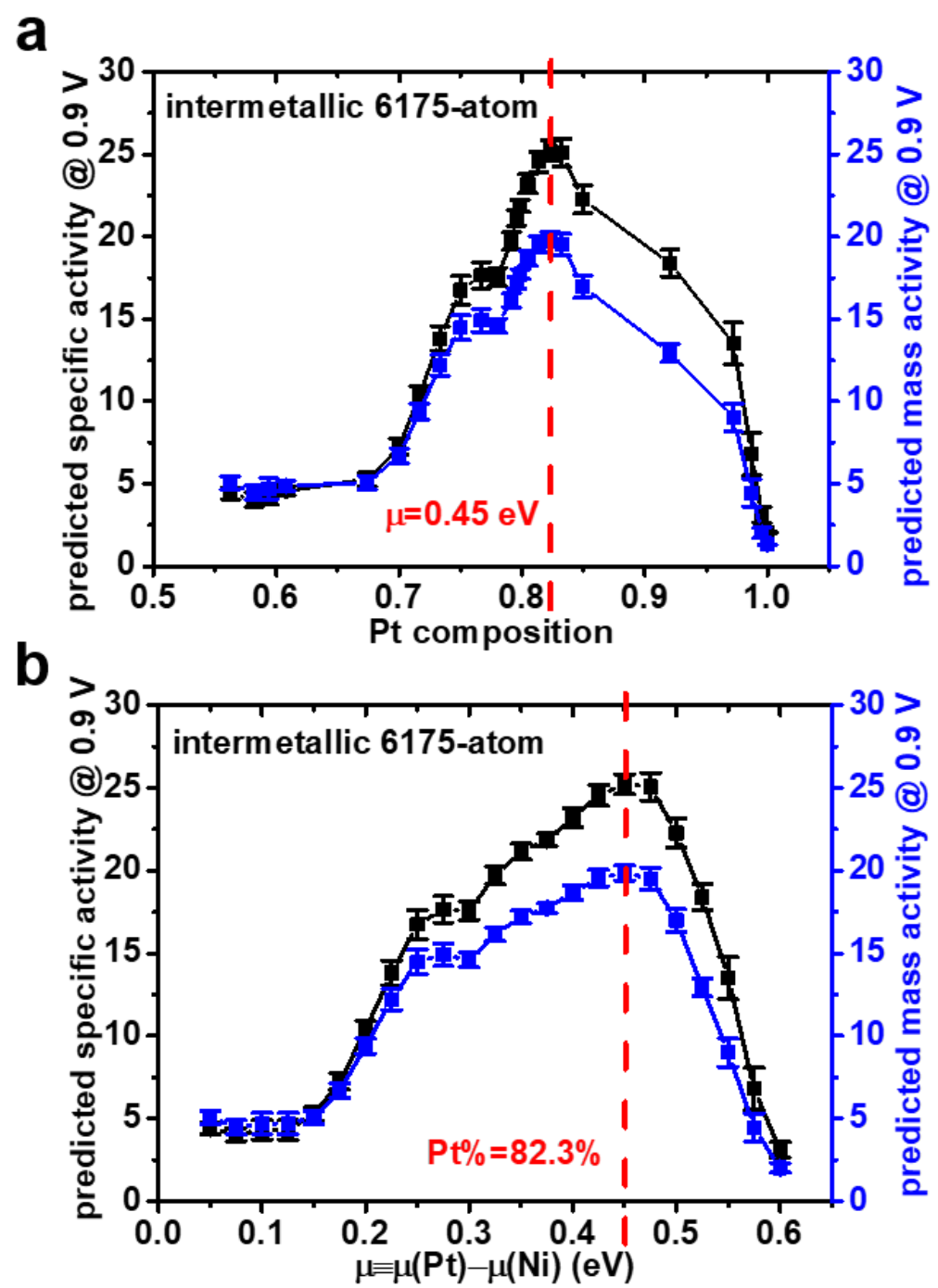

Supplementary Figure 13. The predicted specific and mass activities of intermetallic octahedral nanoparticles with an edge length of $5.5 \mathrm{~nm}$ (6175 atoms) as a function of (a) $\mathrm{Pt}$ composition and (b) chemical potential difference between $\mathrm{Pt}$ and $\mathrm{Ni}$ $(\mu \equiv \mu(\mathrm{Pt})-\mu(\mathrm{Ni}))$. The chemical potential window where bulk $\mathrm{Pt}_{3} \mathrm{Ni}$ is stable is $\mu \in[0.04,0.5] \mathrm{eV}$, which was predicted based on the Pt-Ni-Vacancy cluster expansion in section 1.2 . 

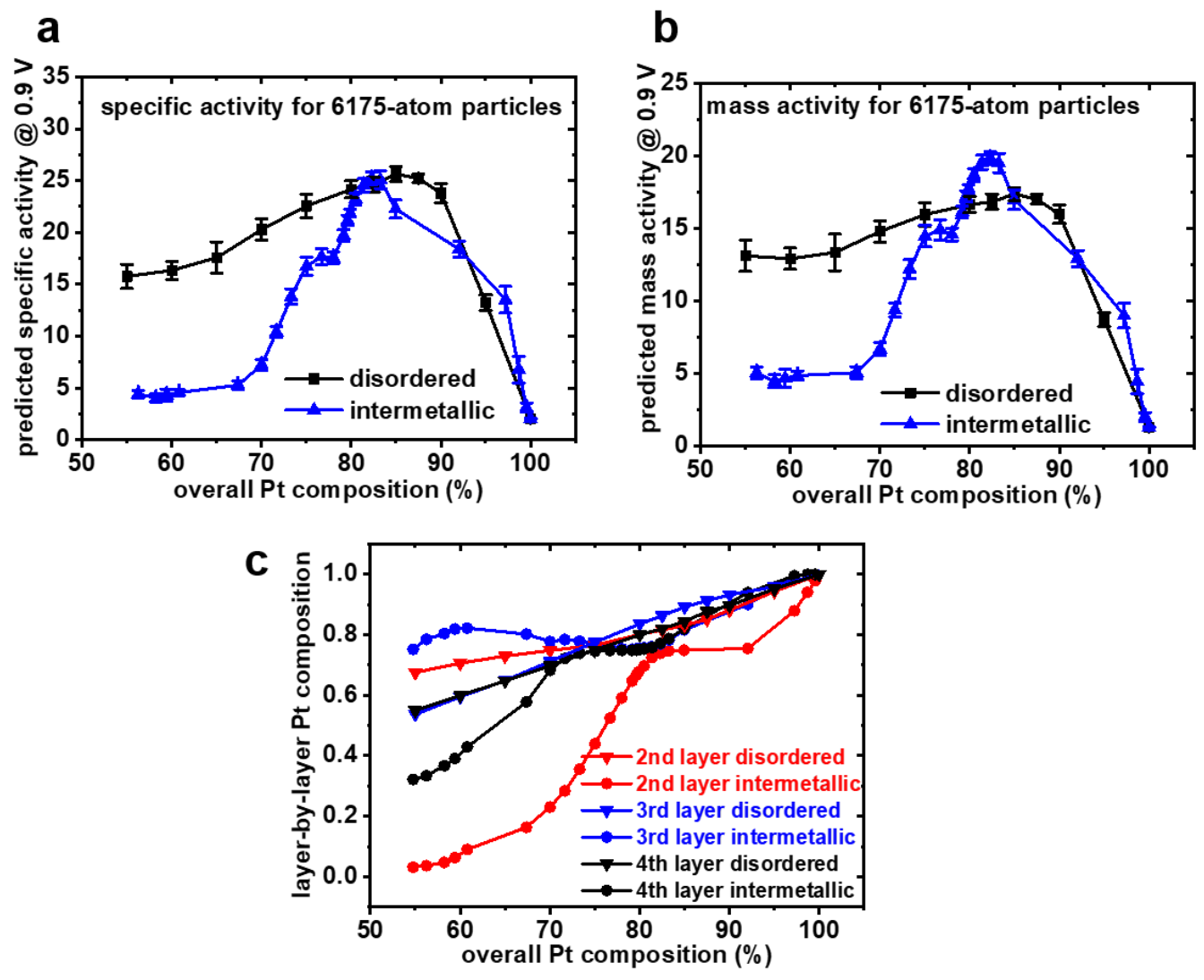

Supplementary Figure 14. The predicted (a) specific activity, (b) mass activity, and (c) layer-by-layer compositions as a function of overall $\mathrm{Pt}$ composition for 6175-atom activated disordered and intermetallic nanoparticles. The "6175-atom" for disordered nanoparticles refers to the pre-activated number of total $\mathrm{Pt}$ and $\mathrm{Ni}$ atoms. 
Supplementary Table 7. Layer-by-layer Pt compositions vs. overall Pt composition for 6175-atom disordered and intermetallic nanoparticles. The 6175 atoms for disordered nanoparticles are the pre-activated number of total $\mathrm{Pt}$ and $\mathrm{Ni}$ atoms.

\begin{tabular}{|c|c|c|c|c|c|c|c|}
\hline \multicolumn{7}{|c|}{ Pt composition (\%) } \\
\hline \multicolumn{7}{|c|}{6175 -atom intermetallic nanoparticles } & \multicolumn{3}{|c|}{6175 -atom disordered nanoparticles } \\
\hline overall & $2^{\text {nd }}$ layer & $3^{\text {rd }}$ layer & $4^{\text {th }}$ layer & overall & $2^{\text {nd }}$ layer & $3^{\text {rd }}$ layer & $4^{\text {th }}$ layer \\
\hline 54.76 & 3.17 & 75.15 & 32.14 & 55 & 67.55 & 53.71 & 54.95 \\
\hline 56.24 & 3.64 & 78.51 & 33.37 & 60 & 70.60 & 59.49 & 59.92 \\
\hline 58.22 & 4.71 & 80.42 & 36.67 & 65 & 72.95 & 64.80 & 64.77 \\
\hline 59.41 & 6.28 & 81.89 & 39.07 & 70 & 74.83 & 71.19 & 69.76 \\
\hline 60.79 & 9.00 & 82.18 & 42.90 & 75 & 76.55 & 77.52 & 74.94 \\
\hline 67.38 & 16.30 & 80.21 & 57.79 & 80 & 80.06 & 83.59 & 79.98 \\
\hline 70.00 & 23.01 & 77.76 & 68.26 & 85 & 83.18 & 89.22 & 84.42 \\
\hline 71.66 & 28.37 & 78.39 & 72.16 & 90 & 88.11 & 93.25 & 89.65 \\
\hline 73.31 & 35.50 & 77.87 & 73.88 & 95 & 94.31 & 95.84 & 95.14 \\
\hline 75.02 & 43.98 & 76.81 & 74.56 & 100 & 100 & 100 & 100 \\
\hline 76.69 & 52.46 & 75.88 & 74.80 & & & & \\
\hline 78.00 & 59.07 & 75.39 & 74.84 & & & & \\
\hline 79.16 & 64.73 & 75.16 & 74.84 & & & & \\
\hline 79.60 & 66.57 & 75.21 & 74.91 & & & & \\
\hline 79.87 & 67.67 & 75.34 & 74.98 & & & & \\
\hline 80.48 & 69.72 & 75.69 & 75.32 & & & & \\
\hline 81.36 & 72.49 & 76.03 & 75.74 & & & & \\
\hline 82.29 & 73.82 & 76.95 & 77.16 & & & & \\
\hline 83.25 & 74.63 & 78.19 & 78.70 & & & & \\
\hline 84.95 & 74.88 & 81.61 & 81.93 & & & & \\
\hline 92.05 & 75.48 & 90.05 & 93.92 & & & & \\
\hline 97.23 & 87.90 & 99.40 & 99.50 & & & & \\
\hline
\end{tabular}




\section{Layer-by-layer atomic structures of intermetallic Pt-Ni nanoparticles}

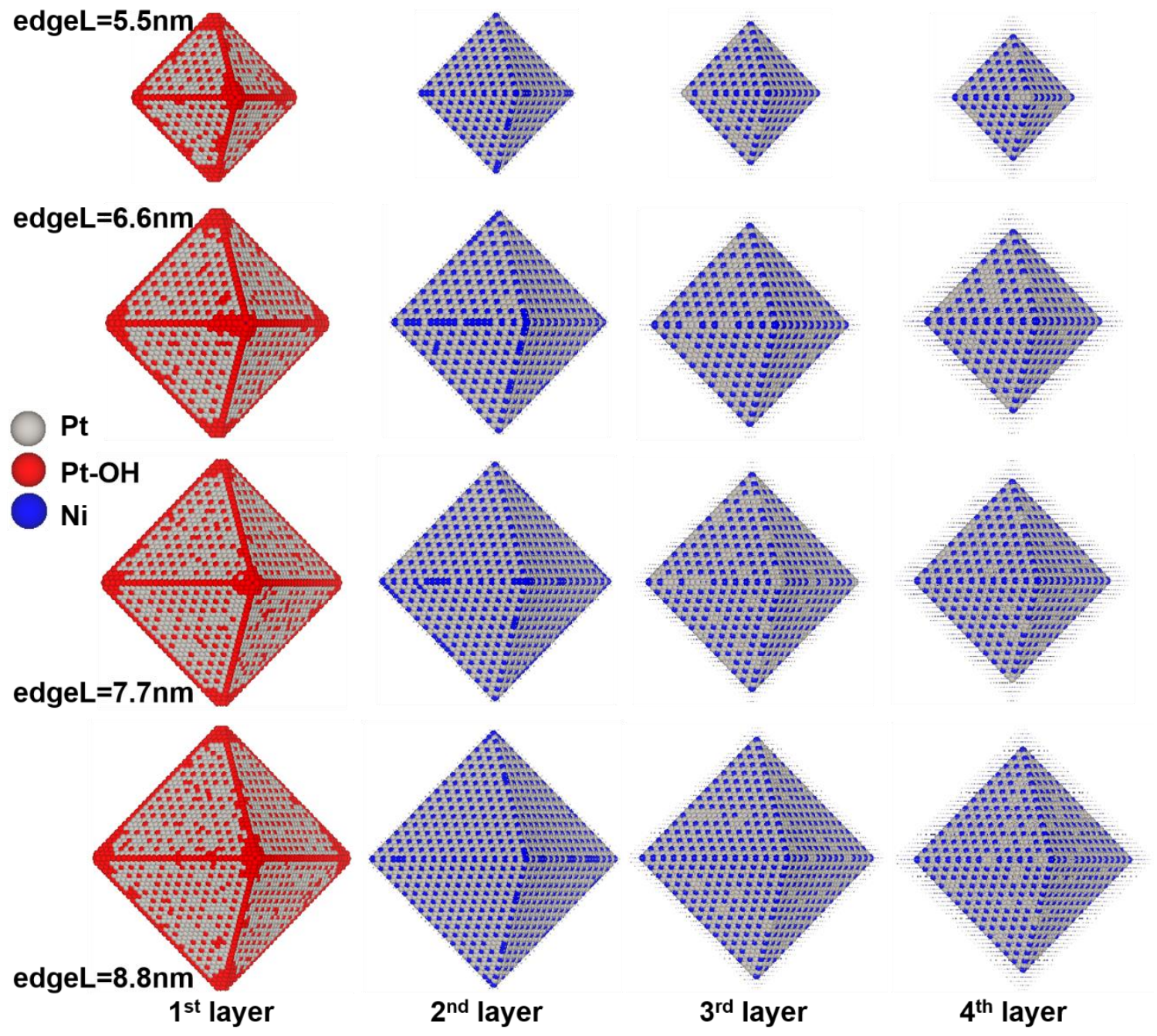

Supplementary Figure 15. The layer-by-layer atomic structures of representative snapshots at $298 \mathrm{~K}$ for intermetallic $\mathrm{Pt}-\mathrm{Ni}$ nanoparticles at $\mu=0.45 \mathrm{eV}$ with adsorbed $* \mathrm{OH}$ determined by KMC run at an edge length of $5.5 \mathrm{~nm}, 6.6 \mathrm{~nm}, 7.7 \mathrm{~nm}$, and $8.8 \mathrm{~nm}$. There exists an $\mathrm{L}_{2}$ structure on subsurface layers. 


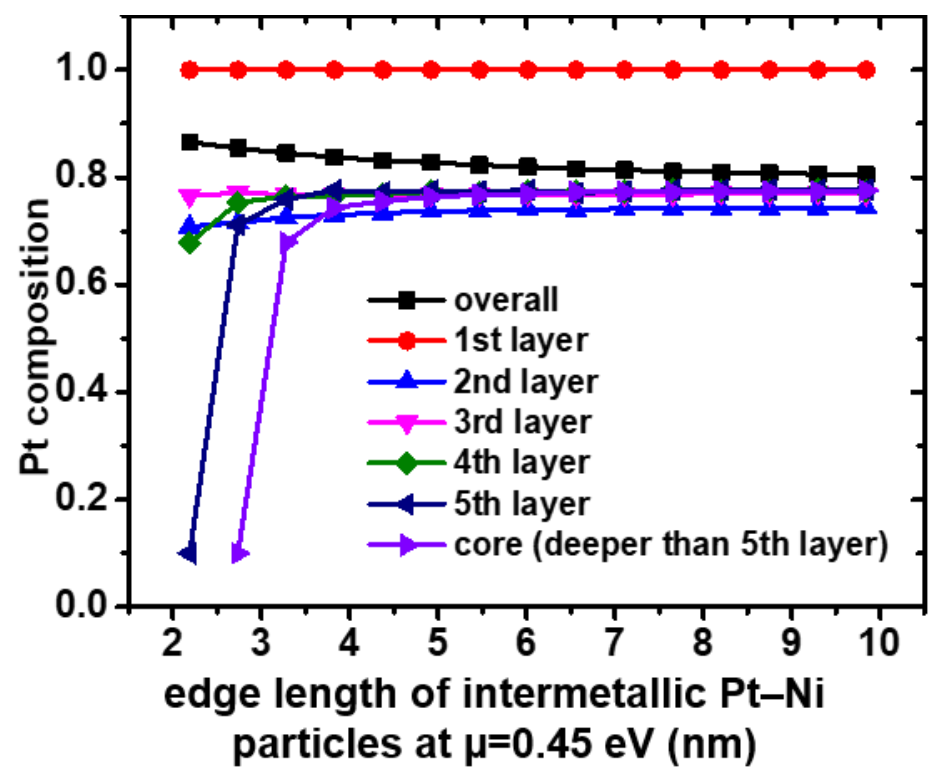

Supplementary Figure 16. The layer-by-layer Pt composition for intermetallic $\mathrm{Pt}-\mathrm{Ni}$ nanoparticles at $\mu=0.45 \mathrm{eV}$ as a function of particle edge length. 
Supplementary Table 8. The predicted layer-by-layer Pt composition for intermetallic PtNi nanoparticles at $\mu=0.45 \mathrm{eV}$ at $298 \mathrm{~K}$ as a function of edge length from $2.2 \mathrm{~nm}$ to $9.9 \mathrm{~nm}$.

\begin{tabular}{|c|c|c|c|c|c|c|}
\hline \multirow{2}{*}{$\begin{array}{l}\text { size in edge } \\
\text { length }(\mathrm{nm})\end{array}$} & \multicolumn{6}{|c|}{ Pt composition (\%) } \\
\cline { 2 - 7 } & overall & $1^{\text {st }}$ layer & $2^{\text {nd }}$ layer & $3^{\text {rd }}$ layer & $4^{\text {th }}$ layer & $5^{\text {th }}$ layer \\
\hline 2.19 & 86.6 & 100 & 70.8 & 76.5 & 67.8 & 10 \\
\hline 2.73 & 85.4 & 100 & 71.6 & 77.1 & 75.3 & 71.1 \\
\hline 3.28 & 84.5 & 100 & 72.6 & 76.8 & 76.5 & 75.9 \\
\hline 3.83 & 83.8 & 100 & 73.0 & 76.8 & 76.6 & 77.6 \\
\hline 4.37 & 83.1 & 100 & 73.4 & 76.6 & 76.9 & 77.2 \\
\hline 4.92 & 82.8 & 100 & 73.7 & 76.9 & 77.4 & 77.5 \\
\hline 5.47 & 82.3 & 100 & 73.8 & 76.9 & 77.2 & 77.4 \\
\hline 6.01 & 82.0 & 100 & 74.1 & 77.0 & 77.4 & 77.6 \\
\hline 6.56 & 81.6 & 100 & 73.9 & 76.7 & 77.4 & 77.1 \\
\hline 7.11 & 81.4 & 100 & 74.2 & 76.8 & 77.3 & 77.4 \\
\hline 7.66 & 81.1 & 100 & 74.2 & 76.8 & 77.6 & 77.6 \\
\hline 8.20 & 81.0 & 100 & 74.2 & 77.0 & 77.6 & 77.6 \\
\hline 8.75 & 80.8 & 100 & 74.2 & 77.0 & 77.6 & 77.8 \\
\hline 9.30 & 80.6 & 100 & 74.3 & 77.1 & 77.6 & 77.6 \\
\hline 9.84 & 80.5 & 100 & 74.4 & 77.0 & 77.5 & 77.7 \\
\hline
\end{tabular}


16. Mass activity of disordered and intermetallic nanoparticles as a function of size

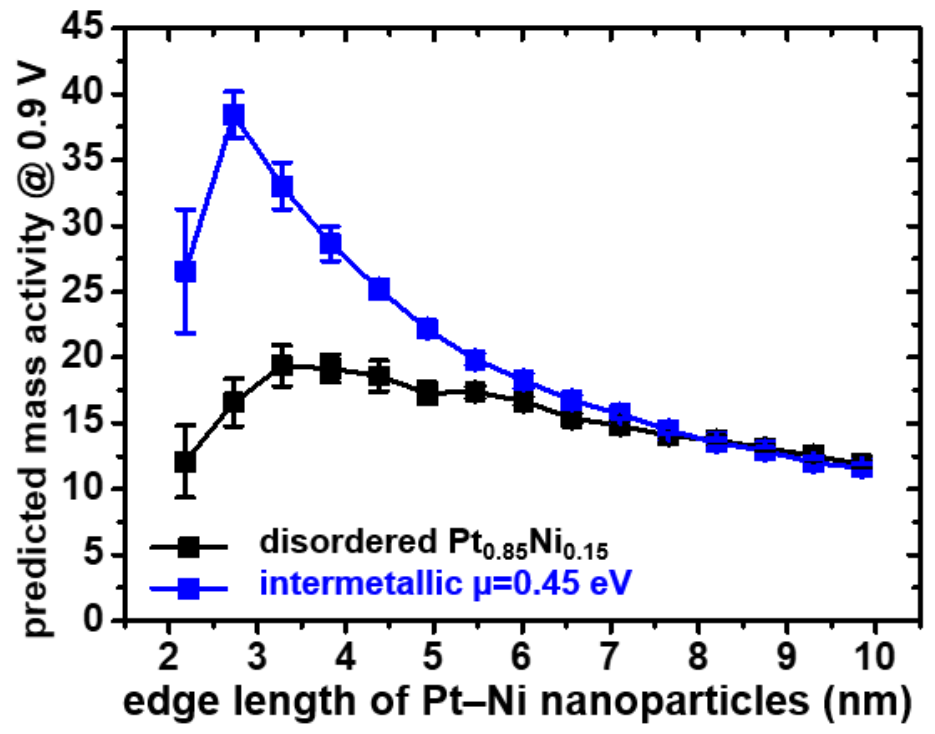

Supplementary Figure 17. Predicted mass activity of disordered $\mathrm{Pt}_{0.85} \mathrm{Ni}_{0.15}$ nanoparticles and intermetallic $\mathrm{Pt}-\mathrm{Ni}$ nanoparticles at $\mu=0.45 \mathrm{eV}$ at $298 \mathrm{~K}$ as a function of edge length. The mass activity values are referenced to that of simulated commercial $\mathrm{Pt} / \mathrm{C}$. The corresponding predicted specific activities are provided in Fig. 2c. 
17. Average * $\mathrm{OH}$ binding energies on (111) sites with $\mathrm{CN}=9$ for disordered $\mathbf{P t}_{0.85} \mathbf{N i}_{0.15}$ and intermetallic Pt-Ni nanoparticles

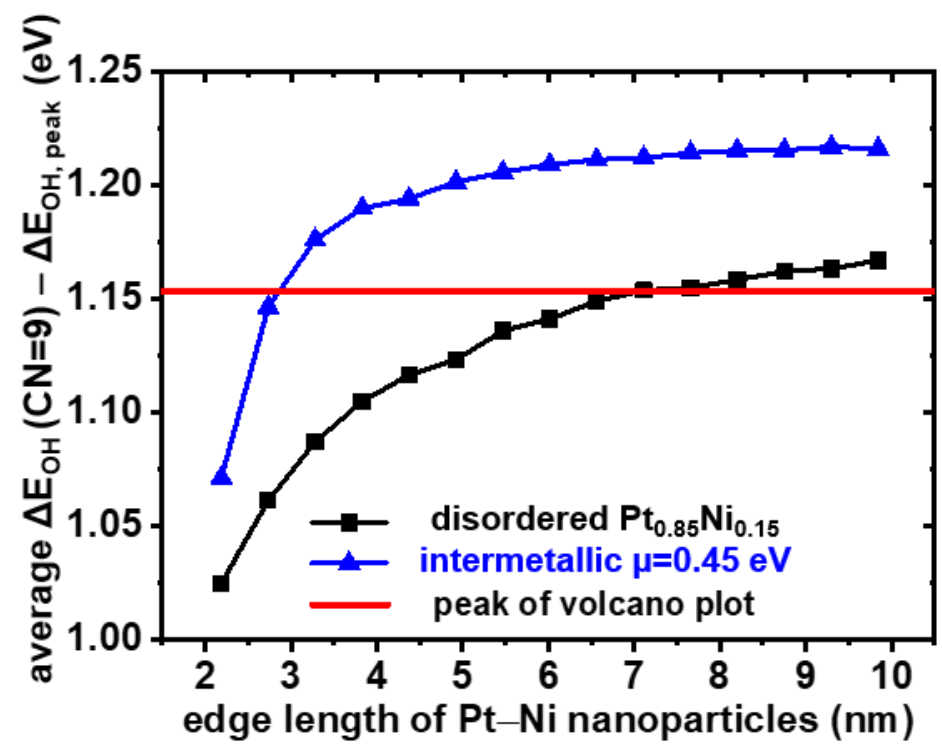

Supplementary Figure 18. Average $* \mathrm{OH}$ binding energies on (111) sites with $\mathrm{CN}=9$ for disordered $\mathrm{Pt}_{0.85} \mathrm{Ni}_{0.15}$ nanoparticles and intermetallic $\mathrm{Pt}-\mathrm{Ni}$ nanoparticles at $\mu=0.45 \mathrm{eV}$ as a function of particle size (edge length). The horizontal red line indicates the volcano plot peak. 


\section{Distribution of $\mathrm{OH}$ binding energy and turnover frequencies}
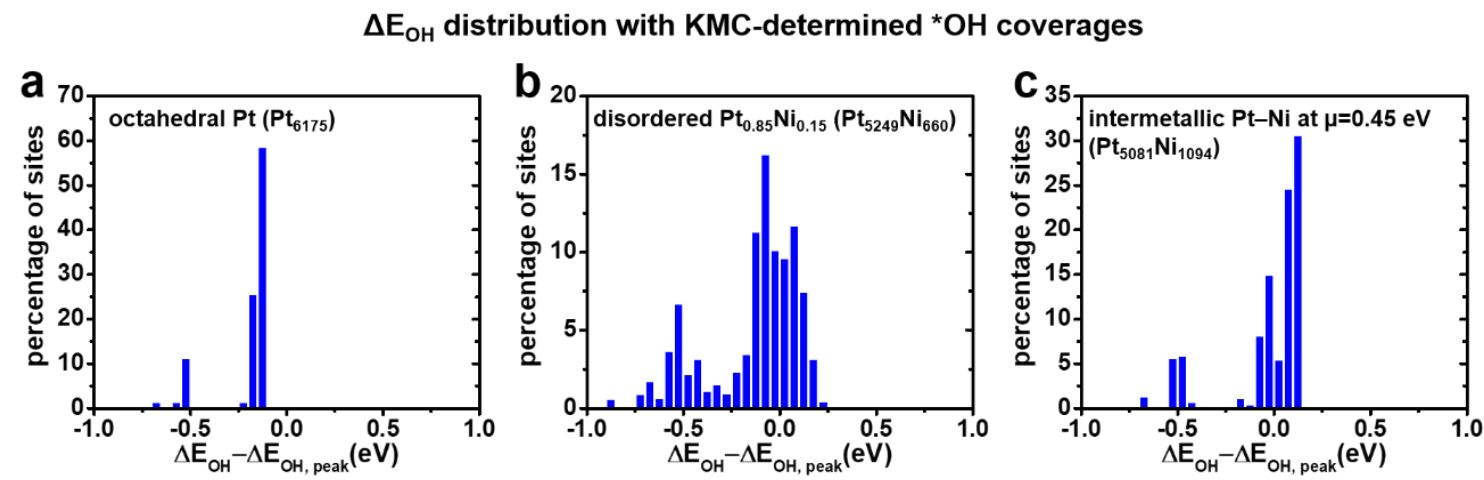

Supplementary Figure 19. Under the KMC-determined $* \mathrm{OH}$ coverages, the histograms showing the distribution of average $\Delta E_{\mathrm{OH}}$ on the surface of three representative nanoparticles in Fig. 4a-c: (a) $\mathrm{Pt}$, (b) disordered $\mathrm{Pt}_{0.85} \mathrm{Ni}_{0.15}$ nanoparticles, and (c) intermetallic $\mathrm{Pt}-\mathrm{Ni}$ nanoparticles at $\mu=0.45 \mathrm{eV}$, respectively. The widths of distribution of $\Delta E_{\mathrm{OH}}$ in figures (a-c) are $[-1.0,1.0] \mathrm{eV}$. 

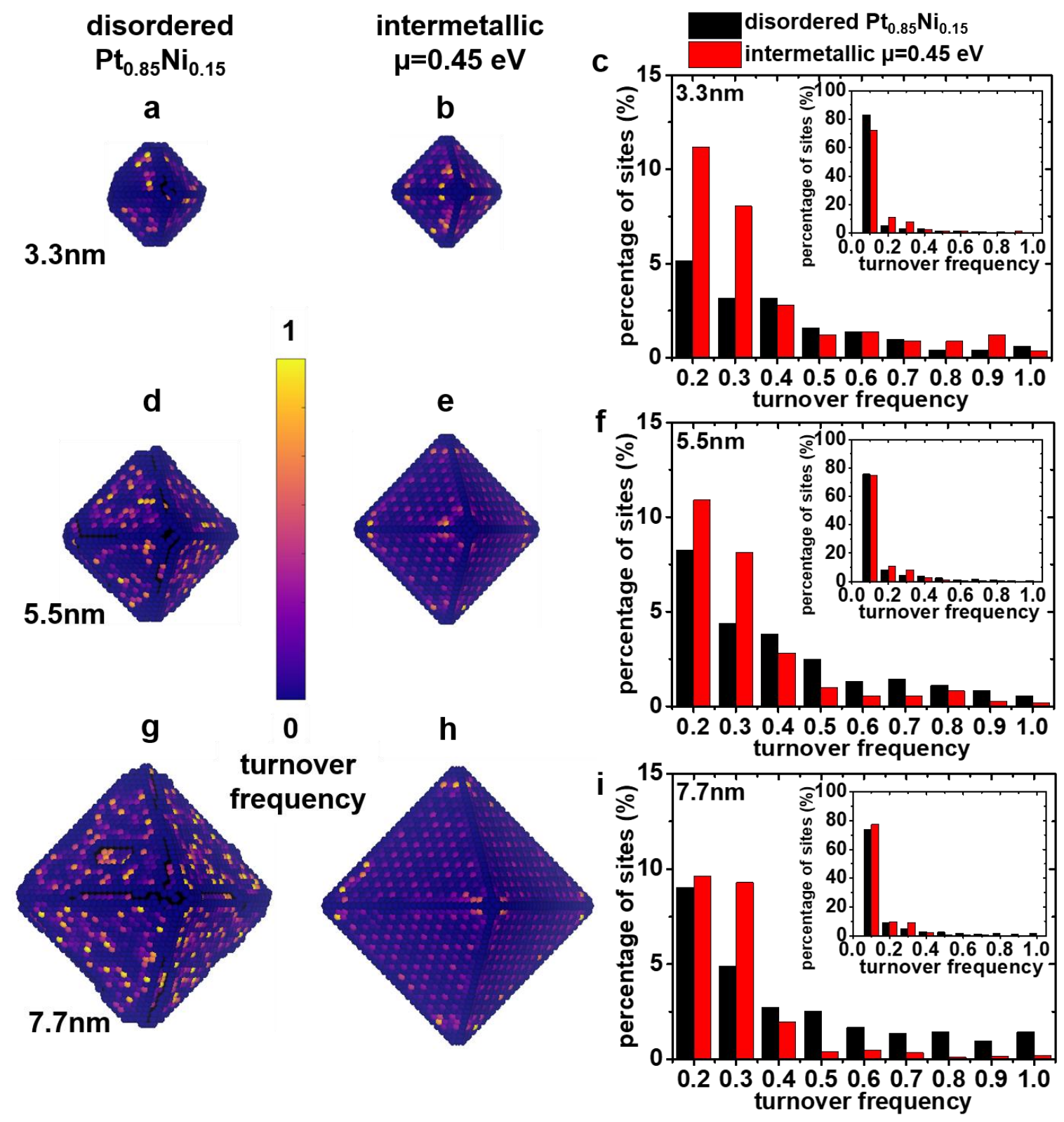

Supplementary Figure 20. Under the KMC-determined *OH coverages, the site-specific average turnover frequency for each surface site of $(\mathrm{a}, \mathrm{d}, \mathrm{g})$ disordered $\mathrm{Pt}_{0.85} \mathrm{Ni}_{0.15}$ nanoparticles and (b, e, h) intermetallic Pt-Ni nanoparticles at $\mu=0.45 \mathrm{eV}$ in an edge length of (a, b) $3.3 \mathrm{~nm}$, (d, e) $5.5 \mathrm{~nm}$, and (g, h) $7.7 \mathrm{~nm}$, respectively. (c, f, i) The corresponding histograms of average turnover frequencies. Both the kinetic Monte Carlo simulations to determine the disordered particles and Metropolis Monte Carlo simulations to determine intermetallic particles were run at 298K. Figures d and e are the same as Fig. 4e, f. 


\section{Reference}

1. Ising E. Beitrag zur Theorie des Ferromagnetismus. Zeitschrift für Physik 1925, 31(1): 253-258.

2. Sanchez JM. Cluster Expansion and the Configurational Theory of Alloys. Phys Rev B 2010, 81: 224202.

3. Sanchez JM, Ducastelle F, Gratias D. Generalized Cluster Description of Multicomponent Systems. Phys A (Amsterdam, Neth) 1984, 128(1-2): 334-350.

4. Huang X, Zhao Z, Cao L, Chen Y, Zhu E, Lin Z, et al. High-performance transition metaldoped Pt3Ni octahedra for oxygen reduction reaction. Science 2015, 348(6240): $1230-$ 1234.

5. Cao L, Mueller T. Theoretical Insights into the Effects of Oxidation and Mo-Doping on the Structure and Stability of Pt-Ni Nanoparticles Nano Lett 2016, 16(12): 7748-7754.

6. Jia Q, Zhao Z, Cao L, Li J, Ghoshal S, Davies V, et al. Roles of Mo Surface Dopants in Enhancing the ORR Performance of Octahedral PtNi Nanoparticles. Nano Lett 2018, 18(2): 798-804.

7. Mueller T, Ceder G. Exact Expressions for Structure Selection in Cluster Expansions. Phys Rev B 2010, 82(Copyright (C) 2015 American Chemical Society (ACS). All Rights Reserved.): 184107.

8. Norskov JK, Rossmeisl J, Logadottir A, Lindqvist L, Kitchin JR, Bligaard T, et al. Origin of the Overpotential for Oxygen Reduction at a Fuel-Cell Cathode. J Phys Chem B 2004, 108: $17886-17892$.

9. Mueller T, Ceder G. Bayesian Approach to Cluster Expansions. Phys Rev B 2009, 80: 024103.

10. Van de Walle A, Ceder G. Automating First-Principles Phase Diagram Calculations. J Phase Equilib 2002, 23: 348-359.

11. Cao L, Zhao Z, Liu Z, Gao W, Dai S, Gha J, et al. Differential Surface Elemental Distribution Leads to Significantly Enhanced Stability of PtNi-Based ORR Catalysts. Matter 2019, 1(6): 1567-1580. 
12. Calle-Vallejo F, Martínez JI, García-Lastra JM, Sautet P, Loffreda D. Fast Prediction of Adsorption Properties for Platinum Nanocatalysts with Generalized Coordination Numbers. Angew Chem, Int Ed 2014, 53(32): 8316-8319.

13. Calle-Vallejo F, Tymoczko J, Colic V, Vu QH, Pohl MD, Morgenstern K, et al. Finding optimal surface sites on heterogeneous catalysts by counting nearest neighbors. Science 2015, 350(6257): 185.

14. Karlberg GS, Rossmeisl J, Norskov JK. Estimations of Electric Field Effects on the Oxygen Reduction Reaction based on the Density Functional Theory. Phys Chem Chem Phys 2007, 9: 5158-5161.

15. Rossmeisl J, Logadottir A, Norskov JK. Electrolysis of Water on (Oxidized) Metal Surfaces. Chem Phys 2005, 319(Copyright (C) 2015 American Chemical Society (ACS). All Rights Reserved.): 178-184.

16. Cao L, Mueller T. Rational Design of $\mathrm{Pt}_{3} \mathrm{Ni}$ Surface Structures for the Oxygen Reduction Reaction. J Phys Chem C 2015, 119(31): 17735-17747.

17. Cao L, Niu L, Mueller T. Computationally generated maps of surface structures and catalytic activities for alloy phase diagrams. Proc Natl Acad Sci U S A 2019, 116(44): 22044-22051.

18. Rossmeisl J, Karlberg GS, Jaramillo T, Norskov JK. Steady State Oxygen Reduction and Cyclic Voltammetry. Faraday Discuss 2008, 140: 337-346.

19. Viswanathan V, Hansen HA, Rossmeisl J, Nørskov JK. Unifying the 2e- and 4e- Reduction of Oxygen on Metal Surfaces. J Phys Chem Lett 2012, 3(20): 2948-2951.

20. Viswanathan V, Hansen HA, RossmeisI J, Nørskov JK. Universality in Oxygen Reduction Electrocatalysis on Metal Surfaces. ACS Catal 2012, 2(8): 1654-1660.

21. Schulze TP. Efficient kinetic Monte Carlo simulation. J Comput Phys 2008, 227(4): 24552462.

22. Serebrinsky SA. Physical Time Scale in Kinetic Monte Carlo Simulations of ContinuousTime Markov Chains. Phys Rev E 2011, 83(3): 037701.

23. Li C, Raciti D, Pu T, Cao L, He C, Wang C, et al. Improved Prediction of Nanoalloy Structures by the Explicit Inclusion of Adsorbates in Cluster Expansions. J Phys Chem $C$ 2018, 122(31): 18040-18047. 
24. Gasteiger HA, Kocha SS, Sompalli B, Wagner FT. Activity Benchmarks and Requirements for Pt, Pt-Alloy, and non-Pt Oxygen Reduction Catalysts for PEMFCs. Appl Catal, B 2005, 56(Copyright (C) 2015 American Chemical Society (ACS). All Rights Reserved.): 9-35.

25. Paulus UA, Wokaun A, Scherer GG, Schmidt TJ, Stamenkovic V, Radmilovic V, et al. Oxygen Reduction on Carbon-Supported Pt-Ni and Pt-Co Alloy Catalysts. J Phys Chem B 2002, 106(16): 4181-4191.

26. Stamenkovic VR, Fowler B, Mun BS, Wang G, Ross PN, Lucas CA, et al. Improved Oxygen Reduction Activity on $\mathrm{Pt}_{3} \mathrm{Ni}(111)$ via Increased Surface Site Availability. Science 2007, 315: 493-497.

27. Gan L, Cui C, Heggen M, Dionigi F, Rudi S, Strasser P. Element-Specific Anisotropic Growth of Shaped Platinum Alloy Nanocrystals. Science 2014, 346(6216): 1502-1506.

28. Metropolis N, Rosenbluth AW, Rosenbluth MN, Teller AH, Teller E. Equation of State Calculations by Fast Computing Machines. J Chem Phys 1953, 21(Copyright (C) 2015 American Chemical Society (ACS). All Rights Reserved.): 1087-1092. 